(Mittheilung aus d. physiol. Institut d. kgl. ung. Franz Josef-Universität in Kolozsvár.)

\title{
Ueber die Reizung des Riechorgans durch directe Einwirkung riechender Flüssigkeiten.
}

Von

Dr. Elemér Veress, Assistent des Instituts.

(Mit 2 Textfiguren.)

In h a l t.

I. Einleitung . . . . . . . . . . . . . . . . . . . . . 368

II. Versuche an der Leiche über die Füllung der Nasenhöhle mit Flüssigkeit 370

1. Beschreibung des anatomischen Präparates . . . . . . . . . . 371

2. Versuche über die Möglichkeit der Füllung der Nasenhöhle mit Flüssigkeit und über die Art und Weise der Einführung derselben 375

3. Versuche unter verschiedenen Form- und Maassveränderungen des anatomischen Präparates . . . . . . . . . . 382

III. Versuche an Lebenden . . . . . . . . . . . . . . . . . . . . . 384

1. Körperhaltung während der Versuche . . . . . . . . . 384

2. Die bei den Versuchen verwendeten Stoffe und einige Versuchsbedingungen .................. . . . 389

IV. Zusammenfassung der Versuchsergebnisse und Erwägung der Möglichkeit, im Wasser zu riechen . . . . . . . . . . . . 393

\section{Einleitung.}

Die Richtigkeit des E. H. W e ber'schen Satzes, dass eine Geruchsempfindung nur dann entsteht, wenn das Riechbare mittelst eines Luftstromes dem Geruchsorgane zugeführt wird, dass also riechende Flüssigkeiten als solche in die Nase gebracht, direct nicht riechbar sind, wurde zuerst von $E$. $\operatorname{Aronsohn}^{1}$ ) bestritten.

Aronsohn benutzte zu seinen Versuchen auf $38-44^{\circ} \mathrm{C}$. erwärmtes, $0,6 \%$ iges oder $0,73 \%$ iges $\mathrm{Na}$ Cl-Wasser als Lösungsmittel

1) Experimentelle Untersuchungen zur Physiologie des Geruchs. Arch. f. (Anat. u.) Physiol. 1886 S. 321. 
für die riechenden Stoffe. Die Einführung dieser Lösungen in die Nase geschah in der bei Th. Weber's Nasendouche üblichen Weise, d. h. bei tiefer Vornüberbeugung des Oberkörpers und des Kopfes. Nach Aronsohn muss bei diesen Versuchen der Nasenrücken die tiefste Lage einnehmen; „so wird die eindringende Flüssigkeit die Regio olfactoria unter Wasser setzen" 1 ).

Aronsohn hat seine. Versuche an sich selbst und Anderen mit Nelkenöl, Campher, Eau de Cologne, Cumarin und Vanillin vorgenommen und aus denselben die Schlussfolgerung gezogen, dass Weber's Satz unbaltbar sei.

Demgegenüber halten aber andere Forscher, wie z. B. W. $\mathrm{Nagel}^{2}$ ), Haycraft ${ }^{3}$ ) und $\mathrm{Zwaardemaker}$ ), diese Ansicht Aronsohn's für nicht annehmbar. Nach $\mathrm{Z}$ wa ardemaker ist eine gewisse Vorsicht in der Deutung dieser Versuche nicht nur darum geboten, weil es schwer fällt, die Luft aus Blindsäcken vollständig auszutreiben, sondern auch aus dem Grunde, weil die Flüssigkeit ihren Weg auch durch den Rachen nehmen kann und so die Riechempfindung eventuell durch eine Geschmacksempfindung gestört wird. Dieser Umstand kann wegen der allgemein bekannten leichten Verschmelzung unserer Riech- und Geschmacksempfindungen einen Versuchsfehler ergeben.

Nach Zwaardemaker ist also diese Frage insolange nicht entschieden, als "Leichenversuche uns die technische Ausführbarkeit einer vollkommenen Füllung der Nasenspalte während der Nasendouche" nicht bewiesen haben.

In neuester Zeit hat sich auch N. Vaschide ${ }^{5}$ ) der Ansicht Aronsohn's angeschlossen; er ordnete seine Versuche folgendermaassen an: Die Versuchsperson athmete in der $\mathrm{Th}$. Weber'schen Körperbaltung mit stark gebeugtem Kopf tief ein und athmete durch

1) Siehe diesbezüglich die Anmerkung auf S. 64. in $Z$ waardemaker, Die Physiologie des Geruchs. W. Engelmann, Leipzig 1895.

2) Vergleichend-physiologische und anatomische Untersuchungen über den Geruchs- und Geschmackssinn und ibre Organe mit einleitenden Betrachtungen der allgemeinen vergleichenden Sinnesphysiologie. Biblioth. zool. Herausg. von Dr. R. Leuckart u. Dr. C. Chun. Heft 18, 1 u. 2. Nägele, Stuttgart 1894.

3) The sense of smell. F. A. Schäfer's Textbook of Physiology vol. 2 p. 1256. Young J. Pentland, Edinburg a. London 1900.

4) 1. c.

5) L'expérience de Weber et l'olfaction en milieu liquide. Compt. rend. hebd. de la Soc. de Biol. t. 53 p. 165.1901. 
den Mund aus. Nachdem die Athmungsbewegungen genügend ergiebig geworden, wurde die Nase zugehalten und in die auf Körpertemperatur erwärmte riechende Flüssigkeit getaucht. Jetzt hatte die Versuchsperson bei geschlossenem Mund kräftig einzuathmen. Vaschide meint, dass auf diese Weise die Riechspalte wenigstens $\mathrm{zum} \mathrm{Theil}$ mit Flüssigkeit gefüllt werden könnte. Ueberzeugende anatomische Beweise hierfür finden wir aber anch bei Vaschide nicht. Wohl hat Vaschide die Gestaltung der Nasenhöhle einer jeden Versuchsperson, als einen wichtigen Factor der Versuche, gründlich untersucht, doch hat sich natürlich der Umstand, ob und inwieweit die Füllung der Riechspalte mit Flüssigkeit thatsächlich erfolgte, seiner Beobachtung entzogen.

Bei meinen auf diese Frage bezüglichen Untersuchungen erschien es mir zweckmässig, den an Lebenden anzustellenden Versuchen gewisse Studien am anatomischen Präparat vorangehen zu lassen.

\section{Versuche an der Leiche über die Füllung der Nasenhöhle mit Flüssigkeit.}

Der Zweck dieser Versuche war, unmittelbar zu beobachten, welchen $\mathrm{Weg}$ die in die Nase gebrachte Flüssigkeit in den bei der Th. Weber'schen Nasendouche gebräuchlichen Kopfhaltungen zu nehmen pflegt, um hierdurch eventuell Beweise für die Möglichkeit einer Anfüllung der Riechspalte mit Flüssigkeit erbringen zu können. Auch hoffte ich, unter Berücksichtigung gewisser anatomischer Fingerzeige feststellen zu können, welche Körperhaltung der vollständigen Anfüllung am günstigsten sei.

Die Vorbereitung des Versuchsobjectes habe ich in ungefähr derselben Art vorgenommen wie dies $\mathrm{Zwa}$ wardemaker ${ }^{1}$ ) und Franke ${ }^{2}$ ) taten, als sie den von der in die Nasenhöhle eingezogenen Luft eingeschlagenen Weg beobachteten.

$\mathrm{Z}$ wa ardemaker verfertigte einen Gypsabguss vom Sagittalschnitte der Nasenböhle eines Pferdekopfes, ersetzte die Nasenscheidewand mit einer Glasplatte und sog dann in das Präparat Lampen-

1) L. c. S. $49-51$.

2) Archiv f. Laryngologie und Rhinologie Bd. 1 Heft 2 S. 236. 1893. Siehe auch $\mathrm{Zwa}$ wardemaker, 1. c. S. 52 . 
russ ein, dessen Weg durch das Glas beobachtet werden konnte. Unabhängig von $Z$ wa ardemaker, aber in ganz ähnlicher Weise, verfuhr Franke mit Sagittalschnitten eines menschlichen Kopfes; statt Lampenruss liess er jedoch Tabakrauch durch das Präparat treten.

Ein derart in der Mittellinie sagittal durchgeschnittener Leichenkopf sollte auch zu meinen Versuchen dienen. Zu diesem Ende habe ich den Kopf eines in höherem Alter verstorbenen Mannes nach Entfernung des Gehirns und nach Abtragung des Unterkiefers durchfrieren lassen und dann mit einer feinen Kreissäge in der Mittellinie durchgesägt.

Diese Arbeit war befriedigend gelungen, denn auf der rechten Seite war der grössere Theil der Nasenscheidewand erhalten geblieben, so dass dementsprechend die linke Nasenhöhle freigelegt erschien.

$\mathrm{Zu}$ den Versuchen verwendete ich die rechte Nasenhälfte. Vor Allem entfernte ich nun die Nasenscheidewand und liess von ihr nur einen ca. $3 \mathrm{~mm}$ breiten Saum bei der vorderen, oberen und hinteren Wand der Nasenhöhle bestehen, so dass also von der Scheidewand nur so viel behalten wurde, wie viel erforderlich war, um die in kleinen Mengen in die Nasenhöhle eingeführte Flüssigkeit zu der Riechspalte auf natürlichem Wege geleiten zu können, ohne dass indess die Beobachtung des von der Flüssigkeit eingenommenen Weges die geringste Beeinträchtigung erfahren sollte ${ }^{1}$ ).

1. Beschreibung des anatomischen Präparates.

Das Abtragen des Septums brachte die seitliche Wand der rechten Nasenhöhle sammt den Muscheln zum Vorschein. Da bei den Versuchen bezüglich der Bewegungsrichtung der Flüssigkeit besonders der Bau und die Weite der zur Riechspalte führenden Wege eine wichtige Rolle spielen, war ich darauf bedacht, noch vor der Schrumpfung oder anderen Veränderungen der Weichtheile pünktliche Maasse zu gewinnen.

Das Loos der in die Nase gebrachten Flüssigkeit hängt in erster Linie von der Weite der zum Geruchsorgan führenden Nasengänge

1) Die Ränder dieses stehen gebliebenen Restes der Nasenscheidewand sind in der Textfigur 1 (auf S. 373) an der dünnen Linie erkenntlich, welche der die vordere, obere und hintere Wand der Nasenhöhle bezeichnenden dicken Linie $a b c d e$ parallel läuft. In der Textfigur 2 (auf S. 374) ist dieser Rand mit $r$ bezeichnet. 
ab. Diese Weite ist aus den an Frontalschnitte vorgenommenen Abmessungen ersichtlich.

Von der rechten Nasenhälfte, welche für die späteren Versuche dienen sollte, war ein Frontalschnitt natürlich nicht zu gewinnen; die auf den linken Frontalschnitt bezüglichen Maasse sind jedoch für die rechte Hälfte nicht maassgebend. Eben darum war ich genöthigt, den Abstand der Nasenmuscheln von der Scheidewand, mit Hülfe der Einführung eines Ersatzes, z. B, eines dünnen Glasplättchens, abzumessen.

Vorher war auch noch die Weite jener Rinne an mehreren Punkten ihres Verlaufes zu bestimmen, welche sich zwischen dem stehen gelassenen Theile des Septums und der seitlichen Wand der Nasenböhle befand.

Diese Rinne dient bei gewissen Stellungen der mit den Nasenlöchern nach oben gekehrten Nasenhöhle, so zu sagen, als Bett und Wegweiser für die in die Nasenhöhle in dünnem Strahl eingeführte Flüssigkeit.

Die Frontalabmessungen dieser Rinne babe ich in einer Entfernung von je $2 \mathrm{~mm}$ von der vorderen, hinteren und oberen Wand der Nasenhöhle vorgenommen. An dem Punkte, an welchem eine verticale Linie, gezogen von dem vorderen Ursprung des unteren Randes der unteren Nasenmuschel, die obere Deckwand der Nasenhöhle schneidet (s. Fig. 1 bei $a)^{1}$ ) war die Breite der Rinne $3 \mathrm{~mm}$, etwas weiter rückwärts, an dem Punkte $b$ (Fig. 1), $2 \mathrm{~mm}^{2}$ ). In der Riechspalte (Fig. 1c) betrug die Breite der Rinne, d. b. der frontale Durchmesser der Riechspalte, 1,3 $\mathrm{mm}$.

Hieraus ist ersichtlich, dass, wenn wir eine Flüssigkeit in dünnem Strahl in die nach oben gekehrte Nasenhöhle derart einführen, dass dieselbe überall entlang der vorderen und oberen Wand eindringen muss, diese Flüssigkeit auf ihrem Wege zum Geruchsorgan eine sich allmählich verengernde Rinne zu benützen gezwungen wird. Hinter

1) Zuckerkandl (Normale and pathologische Anatomie der Nasenhöhle Bd. 1 Aufl. 2 S. 90. W. Braumüller, Wien u. Leipzig 1893) theilt die obere Wand der Nasenhöhle in drei Segmente ein: pars nasalis, p. ethmoidalis und p. sphenoidalis. In der Textfigur 1 liegt $a$ noch in der $\mathrm{p}$. nasalis, während $b$ ungefähr an die Grenze zwischen der p. nasalis und p. ethmoidalis zu stehen Kommt.

2) Nach Zu ekerkandl beträgt die Entfernung zwischen dem Septum und der seitlichen Wand der Nasenhöhle an der p. ethmoidalis $2-3 \mathrm{~mm}$. 
Ueber die Reizung des Riechorgans durch directe Einwirkang etc. 373

der Riechspalte, etwa dem Sinus sphenoidalis entsprechend, erweitert sich wieder diese Rinne. Nun sollte noch der engste Theil der Nasenhöhle festgestellt werden. Hierbei sah ich, dass die geringste lichte Weite nicht entlang der obenerwähnten Rinne zu finden war, denn die Nasenhöhle zeigte sich am engsten zwischen dem kappen-

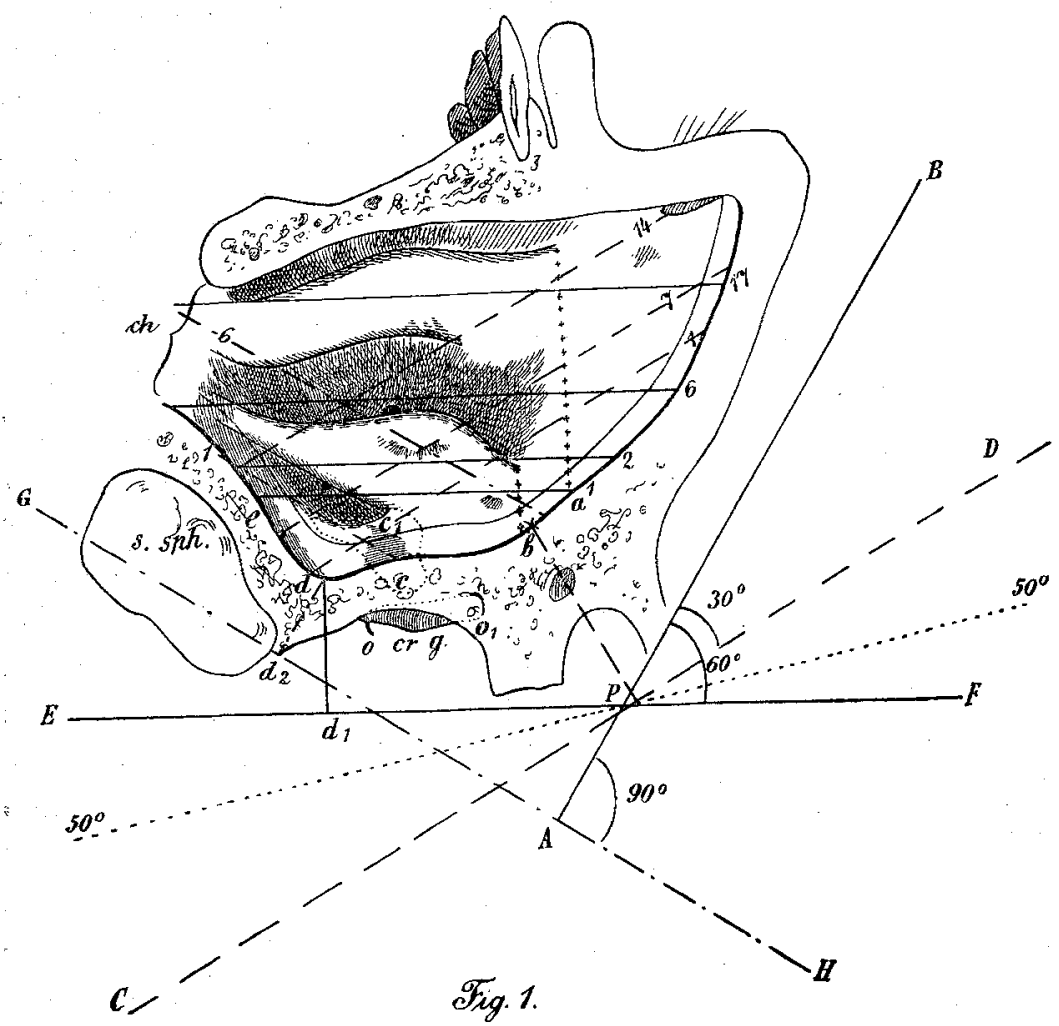

Stellt die Seitenwand der Nasenhöhle in ungefähr natürl. Grösse dar, bezeichnet die. Lage des tiefsten Punktes der Nasenhöhle bei den verschiedenen Kopfstellungen und gibt ein Bild der von den verschiedenen Flüssigkeitsmengen bedeckten Theile der Nasenhöhle. Die in der Nasenhöhle liegenden, parallel zu den Linien $C D, E F$ and $G H$ eingezeichneten Linien stellen dar, einen wie grossen Raum die verschiedenen Flüssigkeitsmengen $(1,7,14 \mathrm{ccm} u$. s.' w.) bei einer Winkelstellung des Präparates von 30,60 und $90^{\circ}$ einnehmen. ch= choana; s. sph. $=$ sinus sphenoidalis ; $c r . g .=$ crista galli; $o-O_{1}=$ Lage des Bulbus olfactorius.

ähnlichen Vorsprung der mittleren Nasenmuschel und dem gegenüberstehenden Theil des Septums. Wie dies aus der Textfigur 2 ersichtlich ist, betrug diese Breite bei dem Punkt $x 1 \mathrm{~mm}$.

Bei Betrachtung des Frontalschnittes muss es besonders auffallen, dass der dritte, der obere Nasengang (Fig. 2, og) tiefer war, 
als es bei Zuckerkand ${ }^{1}$ ) gezeichnet steht, denn dieser Nasengang reichte bis zum obersten Theil der Nasenhöhle ${ }^{2}$ ). Die Folgen dieses Umstandes sollen noch später besprochen werden.

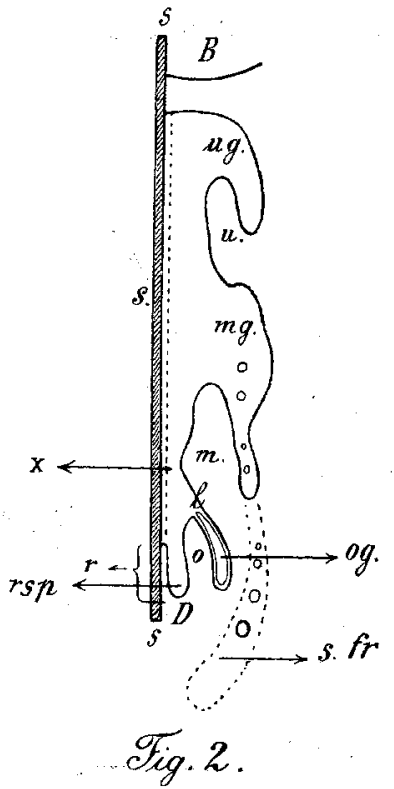

Frontalschnitt der rechten Nasenhälftte, ungefähr in natürl. Grösse, in umgekehrter Lage; auf Grund von Sondirung und directer $\mathrm{Be}$ trachtung gezeichnet. DieSchnittebene liegt am vorderen Ursprung der oberen Nasenmuschel (Verticale $c-c_{1}$ in Fig. 1) $B=$ Boden der Nasenhöhle; $D=$ Dach der Nasenhöhle; $s s=$ Glasplatte (Septum); $u=$ untere Nasenmuschel; $m=$ mittlere; $o=$ obere Nasenmuschel; $u . g .=$ unterer, $m . g .=$ mittlerer, $0 . g .=$ oberer Nasengang; $r s p=$ Riechspalte; $r=$ Rest des Septums; s. $f r .=$ Sinus frontalis, mit Luftbläschen.

1) L. c. Taf. X Fig. 8.

2) In Fig. 1 ist die Ausdehnung dieses sackartigen Ganges mit der punktirten Linie zwischen $c$ und $c_{\mathbf{1}}$ angedeutet.

3) Eine vierte Nasenmuschel kam bei der hier fraglichen Nasenhöhle nicht zur Entwicklung.

4) Beiträge zur mikroskopischen Anatomie der menschlichen Nasenhöhle. Archiv f. mikroskop. Anat. Bd.:39 S. 632. 1892. 
Ueber die Reizung des Riechorganes durch directe Einwirkung etc. 375

greifen kann, wodurch in solchem Falle auch die letztere, zum Theil wenigstens, als Regio olfactoria functioniren könnte. Da ich weder bei v. Brunn, noch bei anderen Autoren diesbezügliche Aufklärungen auffinden konnte, so erschien es geboten, bei meinen Versuchen auch die Verhältnisse der mittleren Muschel zu berücksichtigen.

2. Versuche über die Möglichkeit der Füllung der Nasenböhle mit Flüssigkeit und über die Art und Weise der Einführang derselben.

Um für die geplanten Versuche an Lebenden eine zweckmässige Körperhaltung wählen zu können, musste Rath von der anatomischen Untersuchung geholt werden. Von der Annahme ausgehend, dass der Nasenrücken und die der Riechgegend entsprechende obere Wand der Nasenhöhle unbedingt in einem solchen Verhältnisse zu einander stehen, dass die Stellung des ersteren gewisse Folgerungen auf die Stellung der letzteren zulässt, habe ich die durchschnittlich conforme Richtungslinie des Nasenrückens (Fig. $1 A B$ ) resp. der vorderen Wand der Nasenhöhle zur Richtschnur gewählt.

Die Lage der oberen Wand der Nasenhöhle und dem zu Folge auch die der Riechgegend ändert sich je nach der Grösse des Winkels, welchen die Linie $A B$ mit der horizontalen Ebene bildet. Behufs leichterer Darstellung und Uebersicht der Stellungen zur horizontalen Ebene musste in Fig. 1 die Linie $A B$ als stabil gezeichnet werden und die horizontale Ebene $(C D, E F, G H)$ wechselnde Lagen erbalten. Wenn wir z. B. die Fig. 1 so stellen, dass die Linie $C D$ vor uns horizontal und gleichzeitig in frontaler Ebene liegt, d. h. dass sie mit der Blickrichtung bei primärer Stellung der Augen einen rechten Winkel bildet, so kommt die Nasenhöhle in eine solche Stellung, dass die Linie $A B$ die horizontale Ebene in einem Winkel von $30^{\circ}$ ( $B P D$ ) schneidet. Wenn wir in die soeben besprochene Stellung nicht die Linie $C D$, sondern die Linie $E F$ bringen, so haben wir das Bild der Nasenhöhle bei einer "Winkelstellung" von $60^{\circ}$ u. s. w.

Die Stellung der Nasenhöhle wird bei den versehiedenen "Winkelstellungen" dureh jenen Winkel ( $B P D, B P F$ und $B A H$ ) bestimmt, welcher von der mit dem Nasenrücken durchschnittlich 
parallelen Linie $A B$ und der die Lage der horizontalen Ebene bezeichnenden Linie ( $C D, E F, G H$ ) eingeschlossen wird ${ }^{1}$ ).

$\mathrm{Zu}$ den Versuchen wurde das beschriebene Präparat in folgender Weise vorbereitet: Es wurde zunächst eine Glasplatte ausgeschnitten, deren Form und Grösse der rechten Nasenhälfte annähernd entsprach; diese Glasplatte sollte zum Ersatz des Septums dienen. Da die rechte Hälfte der Nasenhöhle nach dem Durchsägen des Leichenkopfes mit Ausnahme der Choanagegend mit einem 3-10 mm breiten, theils knochigen, theils knorpeligen Saum begrenzt blieb, welcher in seinem ganzen Verlaufe genau in der gleichen Ebene lag, so konnte die zum Verschlusse der Nasenhöhle dienende Glasplatte an die verschiedenen Punkte des Schnittes genau anliegen. Den Saum bestrich ich mit Talg, legte dann die schwach erwärmte Glasplatte an die Stelle des Septums an; der geschmolzene Talg sorgte für vollkommen dichte Schliessung. Die alsdann durch Bespülen mit kaltem Wasser gut abgekühlte Glasplatte blieb in dem wieder erstarrten Talg ziemlich fest haften. Es verblieben demnach nur noch zwei unverschlossene Oeffnungen der Nasenhöhle, nämlich das Nasenloch und die Choana. Wie ich mich später überzeugen konnte, wird die Möglichkeit der Verdrängung der Luft aus der Riechspalte in keiner Weise davon beeinflusst, ob die Choana verschlossen wird oder offen bleibt, da die Luft auch durch das Nasenloch entweichen kann und hier einen hinreichend weiten Weg findet, um der eindringenden Flüssigkeit Platz geben zu können.

Aus diesem Grunde und auch weil bei gewissen Winkelstellungen die Choana verschlossen bleiben muss, habe ich dieselbe zumeist mit Hülfe von mit Talg getränkter Watte verstopft.

Das Präparat wurde dann in eine Universalklemme gefasst, damit es nach den verschiedensten Richtungen bequem bewegt werden könne.

Da zu allererst die Möglichkeit der Anfüllung der Nasenhöhle mit Flüssigkeit zu untersuchen war, habe ich einen Theil des Sep-

1) Die Gestaltung des Nasenrückens ist bei verschiedenen Individuen nicht die gleiche, so dass die diesbezüglich am anatomischen Präparat beobachteten Verbältnisse auf Versuchspersonen nur mit gewissem Vorbehalt anzuwenden sind. Ebenso muss berücksichtigt werden, dass auch das Verhältniss zwischen der Richtung des Nasenrückens und der Lage der oberen Nasenwand wechseln kann, wesshalb es erforderlich ist, dass wir bei jeder Versuchsperson dem Nasenrücken mehrere sich als entsprechend zeigende Lagen zu geben versuchen. 
tums im Präparat stehen gelassen, um die natürlichen Bedingungen, so weit als möglich, beizubehalten.

Es zeigte sich, dass unter solchen Bedingungen die schon erwähnte Rinne (siehe S. 372) bei einer $70^{\circ}$ nicht übersteigenden Winkelstellung als Canal für die Flüssigkeit diente und sie zur Riechspalte führte. Unter normalen Verhältnissen begegnet die Führung der Flüssigkeit in dieser Rinne keinen Hindernissen. Da nu n aber die Riechspalte eigentlich eine directe Fortsetzung des vorderen Teils dieser Rinne darstellt, so ist es natürlich, dass, wiewohl die Riechspalte enger ist als die Rinne bei $a$ und $b$, dieselbe der weiteren Ausbreitung der Flüssigkeit keine Hindernisse in den Weg legt, die Flüssigkeit sowohl vor als auch hinter der Riechspalte das gleiche Niveau einzunehmen strebt, wie das bei communicirenden Röhren zu sehen ist. Die Verengerung der Rinne in der Richtung zur Riechspalte ist nicht nur kein Hinderniss für das Weiterdringen der Flüssigkeit, sondern im Gegentheil, mit Hülfe der Capillarität vertheilt sich die Flüssigkeit sehr rasch in den spaltenartigen Theilen.

Die Verhältnisse würden sich anders gestalten, wenn das Riechorgan in einer sack- oder flaschenartigen Grube liegen möchte. In solchem Falle würde die am Eingang der Höhlung vorbeistürzende Flüssigkeit ein Häutchen bilden und dieses das weitere Eindringen der Flüssigkeit verhindern, gerade so, wie dies geschieht, wenn man eine dünnhalsige Flasche rasch mit Wasser anfüllen will.

Einer ähnlichen Erscheinung kann man übrigens bei der Anfüllung der Nasenhöhle begegnen, jedoch nicht in der Riechspalte, sondern vornehmlich im dritten, obersten Nasengang und im Sinus frontalis. Die obere Nasenmuschel schmiegt sich nämlich der Seitenwand der Nasenhöhle derart an, dass ihr unterer Rand gewissermaassen wie ein Damm sich verhält. Hinter dem vorderen Ursprung der oberen Nasenmuschel wird die Rinne breiter, so dass die Flüssigkeit nicht die ganze lichte Weite derselben auszufüllen gezwungen ist und über den Damm gleiten kann. Es bleibt dem zu Folge im oberen Nasengang meistens eine Luftblase zurück, welche selbst mit' einer Sonde oder mit einem dünnen Draht schwer zu vertreiben ist, da sie den ganzen oberen Nasengang einzunehmen pflegt ${ }^{1}$ ), während,

1) Siehe in Fig. 1 das in der Nähe der Crista galli zwischen $c-c_{1}$ punktirt angedeutete Gebiet. 
die Spalte, aus welcher die Kuppel der Luftblase hervorblickt, gewöhnlich enger ist als der grösste Durchmesser des Bläschens. Je nach der Lage der Nasenhöhle bewegt sich das Luftbläschen im oberen Nasengange hin und her. Die Bildung solcher Luftbläschen ist insbesondere dann wahrnehmbar, wenn die Flüssigkeit in grösserer Menge rasch in die Nasenhöhle eingegossen wird, oder aber, wenn das in Fig. 2 mit $l$ bezeichnete Gebiet mit einer dünnen Schleimschicht überbrückt ist.

Wenn indess das Riechepithel thatsächlich sich nicht bis zum unteren Rande der oberen Nasenmuschel erstreckt, so ist die Bildung: dieser Luftblasen auch dann ohne Belang, wenn sie selbst bei Versuchen an Lebenden in Frage kommt. Vom Riechorgan ist nämlich das Luftbläschen durch die ganze Dicke der oberen Muschel, überdies auch noch durch eine Flüssigkeitsschicht getrennt. In dem zwischen der seitlichen Oberfläche der oberen Muschel und der seitlichen Nasenhöhlenwand befindlichen Theile der Nasenhöhle ist kein Riechepithel vorhanden; die Luftblase ist aber eben hierher eingeschlossen, und selbst wenn sie entweichen sollte, kann sie bei ihrem Aufsteigen das Riechorgan nicht streifen.

Dasselbe gilt auch für die Anfüllung anderer Höhlungen und Taschen. So habe ich öfters beobachten können, dass z. B. auch aus dem Hiatus semilunaris und aus dem Ostium frontale Luftbläschen aufsteigen. Wenn ich bei durchfallendem Licht den Sinus frontalis durch den Schädelgrund beobachtete, konnte ich durch die durchscheinende Schädelwand öfters ganz klar wahrnehmen, dass die Flüssigkeit in den Sinus frontalis eingedrungen ist und die Luft aus demselben in ähnlicher Weise verdrängt hat, wie dies bei mit ihrer Oeffnung nach oben gekehrten, in's Wasser untergetauchten dünnhalsigen Flaschen geschieht. Wenn jedoch die schon erwähnten hindernden Umstände obwalten, so wird der Flüssigkeit das Eindringen nicht immer möglich.

Es ist selbstverständlich, dass, wenn es sich um Versuche an Lebenden handelt, das Resultat derselben gänzlich unbeeinflusst bleibt von dem Umstand, ob Luftbläsehen aus dem Sinus frontalis aufsteigen oder eventuell dort oder in einem anderen vom Riechorgan durch Flüssigkeit getrennten Täschchen eingeschlossen bleiben.

Nach einem jeden, am Präparat vorgenommenen Versuche habe ich die Nasenhöhle sorgfältig entleert und abgewartet, bis der letzte Rest der Flüssigkeit aus den Täschchen und Winkeln der mit 
dem Nasenloch nach unten gekehrten Nasenböhle abgeflossen war. Wird die Nasenhöhle nicht sorgfältig entleert, so sammelt sich die zurückgebliebene Flüssigkeit jm tiefstliegenden Theil der Nasenhöhle; eventuell gerade in der Riechspalte an, und würde bei späteren Versuchen das Vordringen der eingegossenen Flüssigkeit erleichtern.

Des Weiteren sollte nun ermittelt werden, welche Flüssigkeitsmengen bei den verschiedenen Winkelstellungen des Präparates zum vollen Ueberschwemmen des Riechorganes erforderlich sind, ferner, welche Stellen der Nasenhöhle bei den verschiedenen Kopfhaltungen am tiefsten zu liegen kommen, und in Verbindung hiermit, welche Haltung des Kopfes zur Erreichung möglichst bester Resultate an Lebenden die vortheilhafteste ist.

Aus einer Burette oder Pipette habe ich genau abgemessene Flüssigkeitsmengen, je nachdem es zur Erreichung meines Zweckes erforderlich war, in langsamem oder raschem Tempo in die mit der Glasplatte verschlossene Nasenhälfte eingeführt und die: Lage der verschiedenen Flüssigkeitsmengen sofort graphisch aufgenommen (s. Fig. 1). Um den Verlust eines Theiles der Flüssigkeit bei Verwendung grösserer Mengen zu verhindern, habe ich bei diesen Versuchen die Choana stets verstopft.

1. Bei einer Winkelstellung von $0^{\circ}$, d. h. wenn der Nasenrücken in horizontale Lage gebracht war, konnte nur der an der vorderen Wand der Nasenhöhle sich entlang ziehende Theil der Rinne unter Flüssigkeit gesetzt werden. Diese Lage entspricht der Kopfhaltung, bei welcher Aronsohn seine Versuche ausführte, wie er es selbst in seiner Antwort auf die diesbezüglichen Fragen Zwaardemaker's angibt ${ }^{1}$ ). Nach Aronsohn soll die eindringende Flüssigkeit das Riechorgan bedecken, wenn der Nasenrücken in eine solche Lage gebracht wird, dass er den Boden der Nasenhöhle bildet. $\mathrm{Zw}$ a a rdemaker bemerkt hierzu mit vollem Recht, dass unter solchen Umständen das Riechorgan vertical gestellt wird, welche Lage dem Untertauchen nicht zweckdienlich sein kann.

An meinem Präparat hat zwar das Riechorgan selbst bei einer Winkelstellung von $0^{\circ}$ keine verticale Lage eingenommen, doch war die Unvortheilhaftigkeit einer solchen Winkelstellung auch unter diesen Umständen merklich. Wenn wir die Fig. 1 derart vor uns

1) Siehe Zwaardemaker, l. c. S. 64 Anmerkung 2. 
halten, dass die Linie $A B$ horizontal zu liegen komme, und dabei bei primärer Stellung der Augen die Blicklinien in rechtem Winkel schneide, so ist zu ersehen, dass die Flüssigkeit kein höheres Niveau erreichen kann, als den vorderen Ursprung der mittleren Muschel. Ein grösseres Gebiet kann nicht untergetaucht werden, da die Flüssigkeit durch das im Verhältniss zu diesem Punkt tiefer liegende Nasenloch wieder abfliessen muss. Es kann daher auf diese Weise von einer Untertauchung des Riechorganes keine Rede sein.

2. Bei einer Winkelstellung von $30^{\circ}$ (Linie $C D$ horizontal und bei primärer Augenstellung vertical zur Blickrichtung) nahm jener Theil der Rinne die tiefste Stellung ein, welcher in die vom vorderen Ursprung der mittleren Nasenmuschel auf die Linie $C D$ vertical geführte Linie fällt (s. Fig. 1 zwischen $a$ und $b$, beim mit $x$ bezeichneten Punkt). Bei solcher Lage gelang die Füllung der Nasenhöhle ziemlich gut, gleichviel ob das Septum vertical oder etwas nach der Seite geneigt gehalten war. Es kommt manchmal vor, dass zwischen dem Septum und der mittleren Nasenmuschel ein Luftbläschen haften bleibt, doch wird dasselbe durch die eindringende Flüssigkeit immer mehr zu sammengedrückt und platzt schliesslich.

Die Lage der versehiedenen Flüssigkeitsmengen ist in Fig. 1 mit Ḧ̈̈lfe der zur Linie $C D$ parallel gezeichneten punktirten Linien dargestellt. $1 \mathrm{ccm}$ Flüssigkeit genügte zum Ueberschwemmen der oberen Muschel noch nicht, der hintere Theil derselben ragte noch aus der Flüssigkeit heraus. Um die obere Muschel vollständig untertauchen zu können, waren mindestens 10 cem Flüssigkeit nöthig. Wenn die Menge der Flüssigkeit 14 ccm überstieg, so floss der Ueberschuss durch das Nasenloch wieder aus.

3. Bei einer Winkelstellung von $40^{\circ}$ befand sich die tiefstliegende Stelle schon in der Nähe des Riechorganes, und zwar etwas weiter rückwärts als $b$. Die dem Riechorgan entsprechende obere Wand der Nasenhöhle ist, wie bei $30^{\circ}$, doch in geringerem Grade, nach vorne geneigt. $2 \mathrm{ccm}$ Flüssigkeit genügten, um das Riechorgan, 5 cem, um die ganze obere Muschel und den grösseren Theil der mittleren Muschel, und schliesslich $10 \mathrm{ccm}$, um die ganze mittlere Muschel zu überschwemmen. Bei einer grösseren Flüssigkeitsmenge als $20 \mathrm{cem}$ floss der Ueberschuss aus dem Nasenloch heraus, ohne die Choana erreicht zu haben.

4. Bei einer Winkelstellung von $50^{\circ}$ nimmt die obere Wand der Nasenhöhle ungefähr eine horizontale Lage ein. 
5. Bei einer Winkelstellung von $60^{\circ}$ (Linie $E F^{\prime}$ horizontal und bei primärer Augenstellung vertical zur Blickrichtung) neigt die obere Wand der Nasenhöhle meistens etwas nach rückwärts; der tiefste Theil der Nasenhöhle liegt bei $d$. In dieser Stellung konnte die Nasenhöble stets gut gefüllt werden, und $1 \mathrm{ccm}$ Flüssigkeit genügte schon, um die obere Muschel zu verdecken, was unter Berücksichtigung der früher erwähnten Maasse ebenfalls beweist, dass die Leitungsrinne der Flüssigkeit entlang der vorderen, oberen und hinteren Wand der Nasenhöhle in der Riechspalte am engsten ist ${ }^{1}$ ). $6 \mathrm{ccm}$ Flüssigkeit überschwemmten schon die ganze mittlere Nasenmuschel und füllten auch die dahinterliegenden Hiatus und Recessus aus, 17 cem Flüssigkeit überschwemmten die Choana. Wenn die Choana verstopft war, so konnten $22 \mathrm{ccm}$ Flüssigkeit eingeführt werden, bevor ein Ueberschuss aus dem Nasenloch abfloss.

6. Bei einer Winkelstellung von $70^{\circ}$ ist das Dach der Nasenhöhle nach rückwärts geneigt, die tiefste Stelle bleibt noch immer dieselbe wie bei der Winkelstellung von $60^{\circ}$. $1 \mathrm{ccm}$ Flüssigkeit erreichte schon einen kleinen Theil der mittleren Muschel, $6 \mathrm{ccm}$ bedeckten dieselbe schon vollständig; mehr als $6 \mathrm{ccm}$ erreichten die Choana.

7. Bei einer Winkelstellung von $80^{\circ}$ ist das Dach der Nasenhöble noch mehr nach rückwärts geneigt; die tiefste Stelle liegt wie bei der Winkelstellung von $60^{\circ}$. $1 \mathrm{ccm}$ Flüssigkeit hatte die Wirkung wie bei der Winkelstellung von $70^{\circ}$; die Choana wurde sehon von 4 ccm Flüssigkeit erreicht.

8. Bei einer Winkelstellung von $90^{\circ}$ (Linie $G H$ horizontal und bei primärer Augenstellung vertical zur Blickrichtung) ist die Lage des Daches und der tiefsten Stelle der Nasenhöhle wie bei der soeben erwähnten Winkelstellung. $1 \mathrm{ccm}$ Flüssigkeit genügte noch nicht, auch den vorderen Theil der oberen Muschel zu überschwemmen; der vordere Theil des Riechorganes ragte daher aus der Flüssigkeit heraus. $2 \mathrm{ccm}$ Flüssigkeit erreichten schon die Choana, $6 \mathrm{ccm}$ überschwemmten das Riechorgan vollständig, wenn die Choana geschlossen war, doch ragte der vordere Rand der mittleren Muschel noch aus der Flüssigkeit heraus.

1) Siehe diesbezüglich auch das Eindringen der Flüssigkeit bei einer Winkelstellung von $90^{\circ}$ und bei einer Menge von 1 und $6 \mathrm{ccm}$. 
Wenn es die Winkelstellung gestattet, gelangt demnach die Flüssigkeit durch die Rinne zur Riechspalte und füllt dieselbe regelrecht aus. Die Flüssigkeit wird zum Zurücklegen dieses Weges durch physicalische Gesetze, wie das Gesetz der Schwere, der Capillarität und der communicirenden Röhren, gezwungen.

Wenn der Nasenrücken steiler gestellt wird als $75^{\circ}$, so verlässt die Flüssigkeit die beschriebene Rinne, fliesst senkrecht an der Seitenwand der Nasenhöhle herab und erreicht, von der mittleren Muschel in zwei Ströme geschieden, die Choana. Unter solchen Umständen pflegt die Riechspalte von rückwärts, 'von der Choana her, sich anzufüllen, gerade so, als ob wir die Flüssigkeit bei der Choana eingegossen hätten.

Bei Versuchen an Lebenden können also von den aufgezählten Winkelstellungen alle jene, welche $35^{\circ}$ übersteigen, gut verwendet werden, und zwar insbesondere alle jene Winkelstellungen, bei welchen die erwähnte Rinne gewissermaassen als ein zur Riechspalte führender Wegweiser dienen kann $^{1}$ ).

3. Versuche unter verschiedenen Form- und Maassveränderungendes anatomischen Präparates.

Ich hatte nun gewisse Veränderungen in der Gestaltung der Nasenhöhle des Präparates vorzunehmen, um den Einfluss verschiedener anatomischer Verhältnisse prüfen zu können.

Vor Allem habe ich den bisher in einer Breite von $3 \mathrm{~mm}$ erhaltenen Rand des Septums (Fig. $2 r$ ) entfernt. Demzufolge "war nach Wiedereinsetzung der Glasplatte die Weite der Nasenhöhle, der Dicke des entfernten Septumrestes entsprechend, vergrössert; es war daher erforderlich, das Septum in die richtige Entfernung von der Seitenwand zu bringen ${ }^{2}$ ). $\mathrm{Zu}$ diesem Ende habe ich die Glas-

1) Bei meinen Versuchen an Lebenden habe ich zumeist Winkelstellungen von $50^{\circ}$ bis $80^{\circ}$ benützt.

2) Die Glasplatte konnte auch schon bisher die anatomische Lage des Septums (welche in Fig. 2 mit der punktirten Linie angegeben ist) nicht vollkommen nachahmen, so dass das Nasenhöhlenpräparat eigentlich mehr Rauminhalt hatte, als aus den angegebenen, von dem, in seine ursprüngliche Lage zurückversetzt gedachten Septum aufgenommenen Frontalmaassen geschlossen werden könnte. (Die Maasse sind aber selbstverständlich genau, da der Ausgangspunkt bei der Aufnahme derselben die wahre Lage des Septums war.) Der Unterschied im Rauminhalt der Nasenhöhle hat übrigens nur einen geringen 
Ueber die Reizung des Riechorgans durch directe Einwirkung etc. 383 platte, entsprechend der in Fig. 1 von der dicken Grenzlinie umfangenen Fläche, durch Auftragen von Kaliwasserglas derart verdickt, als dies in Fig. 2 mit der punktirten Linie angedeutet erscheint. Hierdurch wurde die Nasenhöhle im erforderlichen Maasse verengt.

An der Seitenwand habe ich die Veränderungen der Einfachheit halber derart bewerkstelligt, dass ich an verschiedenen Stellen geschmolzenen Talg aufpinselte und diese Gebiete, sowie die Wände der ganzen Nasenhöhle, das Septum mitgerechnet, mit einer dicken Salepabkochung oder mit einer zähen Gummilösung überzog, hierdurch gleichfalls eine Verengerung der Nasenhöhle zu erzielen und die bei Lebenden zu findenden Verhältnisse möglichst nachzuahmen trachtend.

Die in der Nasenhöble vorgenonımenen Gestaltsveränderungen, deren einige krankhafte Veränderungen nachahmen sollten, waren im Einzelnen die folgenden:

1. Verschiebung des Ursprunges der mittleren Muschel nach vorne und Verdickung dieser Muschel, wodurch der frontale Abstand des Septums von der Seitenwand sich bedeutend verringerte. Unter solchen Umständen gelingt es leicht, die Nasenhöhle zu füllen.

2. Umgestaltung der Riechspalte $\mathrm{zu}$ einem taschenähnlichen Gang (ungefähr in der Form des oberen Nasenganges, siehe Fig. $2 \circ g$ ) durch Verdickung des unteren Randes der oberen Nasenmuschel. Auch in solchem Falle findet die Flüssigkeit einen Weg zur Riechspalte, und zwar durch Vermittelung der erwähnten Rinne.

3. Kleine Erhöhungen am Boden der Rinne, z. B. entlang der oberen Wand der Nasenhöhle, wodurch der Verlauf der Linie $a d$ in Fig. 1 unregelmässig wellenförmig wird. Selbst diese Verbältnisse verhindern die Anfüllung der Nasenhöhle nicht. Es bildet sich zwar bei gewissen Winkelstellungen, z. B. bei $80-90^{\circ}$, wenn die dammartige Erhöhung des Bodens der Rinne bei $b$ liegt, über diesen Damm beim schnellen Eingiessen der Flüssigkeit ein rascher Abfluss, so zu sagen eine Cascade, unter welcher Luftbläschen sich bewegen. Diese werden indess späterhin durch die von rückwärts, von der Choana her aufsteigende Flüssigkeit verdrängt. Selbst verdoppelte Dämme im Verlaufe der Rinne verursachen keine unüberwindbaren

Einfluss auf die Folgerungen, welche aus dem Ergebniss der bei gewissen Winkelstellungen mit den aufgezählten Flüssigkeitsmengen angestellten Versuche gezogen werden können. 
Schwierigkeiten. In solchen Fällen kann auch die Capillarität dem Vordringen der Flüssigkeit bedeutend zur Hülfe sein.

4. Der die Nasenhöhle überziehende Schleim, welchen ich mit zäher, dicker Gummilösung nachgeahmt habe, kann insofern zu Versuchsfehlern führen, als er Luftbläschen in sich einschliessen kann, z. B. durch Ueberbribckung des Bettes der Rinne. Ein solches, an der Wand der Nasenhöhle haftendes Luftbläschen kann eventuell so lange nicht vertrieben werden, bis der Schleim von der Wand der Nasenhöhle sich nicht ablöst.

\section{Versuche an Lebenden.}

Die hier zu beschreibenden Versuche habe ich an Herrn Cand. med. P. R., Stud. med. J. K. und an mir selbst ausgefübrt, nachdem ich mich vorher überzeugt hatte, dass bei uns allen dreien gute Riechempfindlichkeit in beiden Nasenhöhlen besteht und die anatomischen Verhältnisse normale sind.

\section{Körperhaltung während der Versuche.}

Da der Zweck der Versuche die Untertauchung des Riechorganes war, musste die Riechspalte der am tiefsten liegende Theil der Nasenhöhle werden.

In dieser Hinsicht hat A rons ohn einen Irrtbum begangen, wenn die Kopfhaltung bei seinen Versuchen in der That immer eine derartige war, dass der Nasenrücken den Boden, also den am tiefsten liegenden Theil der Nasenhöhle bildete. Wie bereits erörtert, kann bei einer solchen Kopfhaltung die ganze Riechspalte nicht übersehwemmt werden. Bei tiefer Vornüberbeugung des Oberkörpers kann auch eine derartige Kopfstellung erreicht werden, bei welcher nicht der Nasenrücken, sondern das Riechorgan die tiefste Stelle in der Nasenhöhle einnimmt. Dies ist insbesondere dann der Fall, wenn man das rechte Bein stark nach rückwärts ausstreckt, das linke Knie bengt und den Kopf mit dem Schädeldach nach unten und dem Gesicht nach rückwärts, an die mediale Seite des gebogenen linken Knies anlehnt; in solcher Lage können wir bis $90^{\circ}$ jede beliebige Winkelstellung einnehmen und auch rasch Aenderungen der Kopfhaltung ausführen ${ }^{1}$ ). Diese Körperhaltung wurde zumeist dann in Anspruch

1) Bei an mir selbst angestellten Versuchen habe ich die Winkelstellung mit Hülfe eines Spiegels controlirt. 
genommen, wenn es sich um Versuche nur an der einen Nasenbälfte handelte.

Obwohl in gewissen Stellungen schon $1 \mathrm{ccm}$ Flüssigkeit zur Bedeckung des Riechorganes genügt, habe ich dennoch der grösseren Sicherheit halber stets so viel Flüssigkeit verwendet, dass der Ueberschuss sich aus der Nase ergoss.

Aus der einen Nusenualfte gelangt Flüssigkeit nur dann in die andere, wenn der Nasenrücken eine Winkelstellung von $70-90^{\circ}$ einnimmt; in gewissen Fällen konnte ich also die eine Nasenhälfte zur Controle, mit Hinsicht auf etwa auftretende Ermüdungserscheinungen, unberührt lassen. Wenn dies nicht erforderlich war, habe ich es stets vorgezogen, auch die andere Nasenhälfte zu füllen, damit in dieser letzteren zu Folge der unbehinderten Luftströmung nicht etwa Anlass zu einer Geruchsempfindung gegeben sei.

Da die Stauung des Blutes im Kopfe zu einer. Störung der Beobachtung führen kann, habe ich des öfteren die Versuchspersonen aufgefordert, sich einfach auf ihre Kniee und Hände zu stützen und ihren Kopf zwischen den Armen herabhängen zu lassen, worauf dann die Flüssigkeit aus einer Burette oder einer Pipette in die Nase eingelassen wurde. Bei anderen Versuchen lag wieder die Versuchsperson mit der Brust so auf einem Tisch, dass der Kopf über den Rand des Tisches hervorragte und beliebig abwärts geneigt werden konnte.

Ferner habe ich auch nach Haycraft ${ }^{1}{ }^{1}$ ) Verfahren, jedoch in vereinfachter Weise, Versuche angestellt, und zwar in folgender Art:

Die Versuchsperson lag rücklings auf einer Bank und liess den Kopf am Ende der Bank nach rückwärts herabhängen, so dass die Riechspalte die möglichst tiefste Stellung einnahm. Hierauf wurde aus einer Burette körperwarme physiologische Kochsalzlösung in die Nase eingeführt, bis beide Nasenhälften vollständig angefüllt waren, und das überflüssige Salzwasser, ohne Luftblasen mit sich zu führen, abfloss. Diese, die Nase anfüllende Flüssigkeit habe ich dann mit lauwarmem Salzwasser ersetzt, welches die zu prüfende riechende Substanz gelöst enthielt.

Bei solcher Haltung des Körpers ist es kaum möglich, dem Kopfe eine kleinere Winkelstellung als $75^{\circ}$ zu geben. Dem zu Folge gelangt die Flüssigkeit meistens nicht entlang der oberen Wand der

1) l. c.

E. Płlüger, Archiv für Physiologie. Bd. 95. 
Nasenhöhle zur Riechspalte, sondern fliesst senkrecht zu der Choana und füllt die Nasenhöhle von rückwärts an. Im Uebrigen kann jedoch die Füllung, wie bereits erwähnt, auch auf diese Weise gut erreicht werden.

Es mag noch erwähnt sein, dass diese Kopfhaltung manchen Versuchspersonen sehr unbequem wird, da zu Folge der Reizbarkeit des Gebietes der Choanen resp. des Nasenrachenraumes, unüberwindliche Schluckbewegungen auftreten. Auch mit einer meiner Versuchspersonen konnte ich aus diesem Grunde die Versuche nur in den bei der Nasendouche üblichen Stellungen vornehmen.

Haycraft's Methode darf aus mehreren Gesichtspunkten als entsprechend bezeichnet werden. Ein Vortheil der Methode z. B. besteht darin, dass, da die mit dem Riechstoff beladene Flüssigkeit in die mit physiologischer Salzlösung bereits vollständig angefüllte Nase an Stelle der letzteren gelangt, jene Geruchsempfindung vermieden wird, welche sonst sich zeigen muss, wenn die riechende Flüssigkeit erst die Luft aus der Nase zu verdrängen hat. Andererseits - steigert sich die Concentration der riechenden Flüssigkeit nur allmählich; hierdurch können verschiedene Unannehmlichkeiten, so z. B. das plötzliche Auftreten einer Schmerzempfindung, vermieden werden. Die vorangehende Einfüllung des Salzwassers bietet auch noch den Vortheil, dass die indifferente Flüssigkeit so zu sagen den Weg für die riechende Flüssigkeit vorbereitet. Es unterbleibt auf diese Weise der Kampf zwischen Flüssigkeit und Luft, welcher als solcher schon zu einer Reizung des Riechorgans führen kann.

Die Vermeidung von andauernder Blutstauung ist nicht nur im Interesse der ungestörten Beobachtung, sondern auch aus dem Grunde wünschenswerth, weil bei gesteigerter Blutfülle die Schleimbäute der Nasenhöhle, also auch der Riechspalte einigermaassen anschwellen, und dies in Verbindung mit anderen Umständen das Eindringen der Flüssigkeit eventuell erschweren kann. Haycraft's Methode entspricht auch dieser Forderung.

Es ist ferner zu erwägen, dass die beim Eingiessen der riechenden Flüssigkeit entstehende Geruchsempfindung zu Nachempfindungen führen könnte. Daher habe ich, ausser wenn es sich um Controlversuche handelte, stets zuerst Salzwasser in die Nase gebracht, ohne Rücksicht darauf, bei welcher Körperstellung die Versuche vorgenommen wurden.

Das Salzwasser habe ich immer vorsichtig in kleinen Portionen 
in die Nase eingeführt. Mit der riechenden Flüssigkeit dagegen wurde die Nase bald in langsamem, bald in rascherem Tempo angefüllt, damit auch die Wirkung der Bewegung der Flüssigkeit beobachtet werden könne.

Durch starkes Vornüberbeugen und darauf folgendes Strecken des Oberkörpers habe ich den Kopf bei zugehaltenen Nasenlöchern in grossem Bogen nach vorwärts und dann nach rückwärts bewegt, um die eventuelle Wirkung der auf diese Weise bedingten Bewegungen der Flüssigkeit beobachten zu können.

Damit der in der Riechspalte sitzende Schleim eine voraus unberechenbare Störung nicht ausüben könne, habe ich in vielen Fällen die Nase 1-2 Stunden vor dem eigentlichen Versuch mit $0,925 \%$ iger $\mathrm{NaCl-Lösung} \mathrm{ausgespült.} \mathrm{Die} \mathrm{Nasengänge} \mathrm{wurden} \mathrm{hierdurch} \mathrm{stets}$ freier zugänglich, wie dies auch diesbezüglich gemachte Messungen beweisen. So konnte ich z. B. einmal bei der ersten Anfüllung nur $19 \mathrm{ccm}$, bei der zweiten unmittelbar darauf folgenden Anfüllung dagegen $22 \mathrm{ccm}$ Flüssigkeit in meine beiden Nasenhälften bringen. Bei einer anderen Gelegenbeit betrug die bei einer Winkelstellung von $80^{\circ}$ zur Anfüllung beider Nasenhöhlen und des Nasenrachenraumes erforderliche Menge Flüssigkeit $25 \mathrm{ccm}$, bei der unmittelbar darauffolgenden zweiten Anfüllung dagegen $30 \mathrm{ccm}$.

Was nun die Frage anbelangt, in welchen Fällen ich darauf rechnen konnte, dass die Riechspalte während der Versuche thatsächlich unter die Flüssigkeit getaucht war, habe ich diesbezüglich ausser den aus der Lage des Kopfes sich ergebenden Schlussfolgerungen noch folgende Punkte zur Richtschnur genommen.

1. Wenn die Flüssigkeit das Riechorgan erreicht, empfindet der Beobachter in vielen Füllen ein lebhaftes Berührungsgefühl, ähnlich jenem, welches entsteht, wenn ein empfindlicher Körpertheil gekitzelt wird. Späterhin aber, wenn die Versuche schon mehrere Wochen hindurch wiederholt werden, tritt in Folge der Angewöhnung dieses Gefühl nur mehr selten auf.

2. Nach Einführung einer gewissen Flüssigkeitsmenge habe ich durch die Nase eingeathmet resp. einathmen lassen. Hatte die Flüssigkeit die Riechspalte bereits überschwemmt, so führte das Schnüffeln in dieser Nasenhälfte zu keiner Geruehsempfindung, wenu nämlich die Flüssigkeitsmenge und die Kopfstellung in richtigem Verhältniss zu einander standen. Bei einer Winkelstellung von $20^{\circ}$ und unter den in Fig. 1 abgebildeten anatomischen Verhältnissen 
ähnlichen Bedingungen, kann in die Nasenhöhle nicht so viel Flüssigkeit eingegossen werden, dass das Riechorgan vollständig überschwemmt sei. Das Abfliessen der überflüssigen Flüssigkeitsmenge könnte eventuell den Glauben erwecken, dass die Nasenhöhle bereits vollständig angefüllt ist. Wenn nun die Versuchsperson durch die Nase einathmet, so berühren von der Oberfläche der Flüssigkeit aufsteigende Riechpartikelchen das Geruchsorgan dort, wo es noch nicht überspült ist. Die hierdurch entstehende Geruchsempfindung kann, wenn wir die Athmungshewegungen der Versuchsperson nicht controliren, zu irrigen Schlussfolgerungen führen. Wenn wir dagegen bei einer Winkelstellung von z. B. $60^{\circ}$ nach Einfüllung der riechenden Flüssigkeit schnüffeln und keine Geruchsempfindung wahrnehmen, so ist es offenbar, dass kein einziger Punkt des Riechgebietes aus der Flüssigkeit herausragt.

Ein solches Verfahren gibt indess noch keine Aufklärung darüber, ob nicht etwa unter der Oberfläche der Flüssigkeit ein Schleimteilchen eine Luftblase einschliesst.

3. Um dies zu erkennen, habe ich nach vollständiger Anfüllung der Nasenhöhle bei zugehaltenen Nasenlöchern den Kopf in der beschriebenen Weise bewegt. Die Bewegungen eines Luftbläschens können eventuell eine reine Geruchsempfindung hervorrufen.

Zur Orientirung über die erfolgreiche Anfüllung der Nase dienten noch folgende Erscheinungen:

4. Beim Abfliessen der Flüssigkeit aus der Nase brachte diese öfters auch sehr reinen, durchsichtigen Schleim mit sich, welcher wahrscheinlich aus der Riechspalte und ihrer nächsten Umgebung sich abgelöst hat.

5. Zu Folge der Reizwirkung der riechenden Flüssigkeit pflegt oft ein eigenthümlich zusammengesetztes Gefühl aufzutreten, auf dessen Charakterisirung noch später zurückgegriffen werden soll.

6. Nach mit den riechenden Flüssigkeiten angeführten Versuchen ändert sich die Schärfe der Riechempfindlichkeit gegenüber dem verwendeten Stoff, sowie gegenüber andere gasartige Riechstoffe, in ganz charakteristischer Weise. So konnte ich z. B. bezüglich des Tabakrauches in sehr auffallender Weise constatiren, dass in solchen Fällen, in welchen eine vollständige Anfüllung der Nase mit Sicherheit vorausgesetzt werden konnte, das Aroma verschieden feiner Tabaksorten eine gleichartige Geruchsempfindung hervorrief, welche 
Ueber die Reizung des Riechorgans durch directe Einwirkung etc. 389

am ehesten mit dem Geruche brennender, trockener Weiublätter oder mit dem Geruche verbrannter Maisstengel verglichen werden kann.

2. Die bei den Versuchen verwendeten Stoffe und einige Versuchsbedingungen.

Nachdem ich an mir selbst erfahren habe, dass sowohl die $0,6 \%$ ige, als auch die nach A ronsobn physiologische $0,73 \%$ ige $\mathrm{NaCl-Lösung} \mathrm{unter} \mathrm{gewissen} \mathrm{Umständen} \mathrm{eigenartige} \mathrm{zusammen-}$ ziehende, wenn auch vorübergehend, so doch etwas schmerzliche Empfindungen verursachte, habe ich als Lösungsmittel für die Riechstoffe $0,925 \%$ iges $\left.^{1}\right) \mathrm{NaCl}$-Wasser benützt, welches keine derartigen Empfindungen auslöste.

Die Riechstoffe habe ich in einer solchen Concentration angewendet, dass ihre specifische Wirkung beim Riechen an der Luft ausgesprochen war, dieselben jedoch nach Einführung in die Nase noch $\mathrm{zu}$ ertragen waren.

1. Eau de Cologne ${ }^{2}$, meistens in $2,5 \%$ iger Lösung.

2. Ylang-ylang ${ }^{3}$ ), meistens in $2,5 \%$ iger Lösung.

3. Essbouquet ${ }^{4}$ ), meistens in $2,5 \%$ iger Lösung.

4. Nelkenöl, meistens in $0,01 \%$ iger Lösung.

5. Origanumöl, meistens in $0,025 \%$ iger Lösung.

6. Pfeffermünzöl, meistens in $0,025 \%$ iger Lösung.

7. Campherwasser, durch Schütteln von Campherstücken mit kaltem Wasser und nachberigem Abfiltriren des Wassers dargestellt.

8. Capronsäure, 3-4 Tropfen in $150 \mathrm{ccm}$ Salzwasser.

9. Dieselbe Lösung, unter Zufügung eines Tropfens Piperidin. Diese letztere Flüssigkeit rief eine ziemlich ekelhafte Geruchsempfindung hervor.

Neben der Isotonie und der Riechconcentration der Lösungen kommt auch noch ihre Temperatur als einer der Componenten des zusammengesetzten Reizes in Betracht, wenn nämlich die Temperatur der Lösungen von jener des Riechorganes bedeutend abweicht. Ruft

1) H. J. Hamburger, Ueber die Regelung der osmot. Spannkraft von Flüssigkeiten in Bauch- und Pericardialhöhle. Archiv f. (Anat. u.) Physiol. 1895. S. 305 .

2) Von Joh. Maria Farina in Cöln.

3) Extrait triple von Schimmel \& Co. in Leipzig.

4) Dito. 
die Flüssigkeit eine Kälteempfindung hervor, so kann sie die Geruchsempfindlichkeit abstumpfen und gleichzeitig auch die Beobachtung stören. Es ist daher, wie dies auch Ar ons ohn und Vaschide thaten, erforderlich, die Flüssigkeiten ungefähr auf Körpertemperatur zu erwärmen.

Die Riechstoffe sind bezüglich der Entfaltung ihrer specifischen Wirkung gegen Aenderungen der Temperatur empfindlich. Zwaardemaker ${ }^{1}$ ) empfieblt daher, diesen Umstand als "Temperaturcorrection" bei der Olfactometrie in Rechnung zu ziehen.

Das Wärmen vergrössert die Beweglichkeit der riechenden Partikeln und löst eine lebhaftere Empfindung aus, als eine solche ohne Erwärmen eintreten würde. Diese Zunahme der verschärften Geruchsempfindung entspricht zunächst der Eigenart des verwendeten Riechstoffes, d. h. sie wird unter Bewahrung der Reinheit der Geruchsempfindung schärfer. Ueber einen gewissen Wärmegrad hinaus wird jedoch das Geruchsorgan und seine Umgebung von combinirteren Reizen berührt; es gesellen sich in Folge dessen mehrminder unangenehme Nebengefühle zu der eigentlichen Geruchsempfindung, wobei vornehmlich die Reizung der Nervenapparate des N. trigeminus die Hauptrolle spielt. Der Charakter der Geruchsempfindung ändert sich hiernach hauptsächlich unter dem Einflusse von an Berührungsgefühle erinnernden Reizzuständen. Das Erwärmen lässt also besonders jene Eigenschaften der Riechstoffe in den Vordergrund treten, welche die Nervenapparate des $\mathrm{N}$. trigeminus reizen können.

Heisse Dämpfe von Eau de Cologne rufen in der Nase eine stechende Empfindung hervor. Riechen wir dieselben aus einer gewissen Entfernung, in welcher der Dampf sich bereits abgekühlt hat, so wird wieder der bekannte angenehme Geruch empfunden. Es bedarf daher nur der Abkühlung, um die specifische Wirkung, die Eigenart wieder hervortreten zu lassen.

Auf $+1,+2{ }^{\circ} \mathrm{C}$. abgekühltes Nelkenöl führt zu einer sehr feinen Empfindung etwas säuerlichen Charakters, selbst dann, wenn der abgekühlte Stoff in warmer Luft gerochen wird. Diese Empfindung ist sehr vorübergehend und kann auch nur schwer in der Erinnerung behalten werden. Auf $15-20^{\circ} \mathrm{C}$. erwärmtes Nelkenöl löst eine tiefdringende, vollkommene Geruchsempfindung aus. Auf $46-48^{\circ} \mathrm{C}$. erwärmt, bedingen die Dämpfe ausser dem Nelkenölgeruch auch noch

1) 1. c. S. 124 . 
Ueber die Reizung des Riechorgans durch directe Einwirkung etc. 391

stechende Nebenwirkungen; diese Begleiterscheinungen lassen sich auf den mittleren Nasengang beziehen.

Mentholkrystalle geben bei gewöhnlicher Zimmertemperatur eine angenehme kühlende Empfindung; der geschmolzene Stoff dagegen führt zu einer sehr widerwärtigen, mit Abwehrreflexen einhergehenden brennenden, stechenden Empfindung.

Das Optimum zur Wahrnehmung der specifischen Eigenschaften von Ricchstoffen liegt daher zwischen gewissen Wärmegrenzen, da die Riechstoffe innerhalb dieser Grenzen ihre specifische Wirkung erreichen, resp. bewahren.

Sowohl das Sinnesorgan, als auch die specifischen Eigenschaften der Riechstoffe fordern daher das Einhalten einer entsprechenden Temperatur.

Da eine Temperatur von $32-38^{\circ} \mathrm{C}$. bezüglich der R. olfactoria und der R. respiratoria als indifferent betrachtet werden kann, habe ich die erwähnten Stofte meistens innerhalb dieser Temperaturgrenzen angewendet, umsomehr, da bei diesen Temperaturen die specifischen Eigenschaften der Lösungen von der angegebenen Concentration keine nennenswerthen Veränderungen aufweisen.

Nur die Lösung von Pfeffermünzöl löst schon bei einer Temperatur von $34^{\circ} \mathrm{C}$. sehr intensive Empfindungen aus; zu Beginn des Erwärmens steigert sich noch die kühlende Wirkung, doch treten dabei auch schon bohrende Schmerzempfindungen in den Vordergrund. Bei $45^{\circ} \mathrm{C}$. vermindert sich die kühlende Wirkung bedeutend und lässt die Schmerzempfindung intensiver werden: der Riechstoff wirkt also schon als allgemeiner Nervenreiz, wobei die Thätigkeit des Riechorgans gar nicht mehr wahrgenommen wird. Bei $50^{\circ} \mathrm{C}$. ist die kühlende Wirkung nur mehr an der hinteren Wand des Rachens fühlbar; vorne, in der R. respiratoria, wird diese Empfindung durch den starken Schmerz unterdrückt, resp. latent gemacht. Aus diesen Gründen durfte ich Lösungen des Pfeffermünzöles bei einer höheren Temperatur als $34^{\circ} \mathrm{C}$. nicht verwenden.

Ueber meine Versuche babe ich ein detaillirtes Tagebuch geführt; es sollen hier daraus als Beispiel die auf einen der verwendeten Stoffe, nämlich auf das Eau de Cologne, bezüglichen Aufzeichnungen mitgetheilt werden:

2. Mai 1902. Versuch mit verdünntem Alkohol. (5 Theile $90 \%$ iger Alkohol auf 95 Theile 0,9\% ige NaCl-Lösung.) Nachdem der Alkohol ein Bestandtheil des Eau de Cologne ist, muss derselbe als einer der Componenten der Reiz- 
wirkung der Eau de Cologne-Lösung auch gesondert untersucht werden. Bei vornübergebeugtem Oberkörper und ohne die Nasenhöhle mit indifferenter Salzlösung angefülllt zu haben, in die Nase eingeführt, löst der Alkohol öfters Niessen aus und erweckt von dem Riechen des Alkohols an der Luft stammende, nicht ganz bestimmte Erinnerungen, auf Grund deren jedoch der Alkohol zu erkennen ist.

3. Mai 1902. Versuche mit $5 \%$ iger Ean de Cologne-Lösung. Bei $17^{\circ} \mathrm{C}$. sehr unangenehm, unerträglich; verursacht meistens .Niessen.

Nach 2 Stunden ein weiterer Versuch mit 2,5\% iger Lösung bei $17^{\circ} \mathrm{C}$. Bei vornübergebeugtem Oberkörper in die Nase direct eingeführt, entsteht eine eigenthümliche, zusammengesetzte Empfindung; zur bereits bekannten Wirkung des Alkohols gesellt sich noch ein anderer schwer zu bestimmender Reizzustand des Riechorgans, welcher eine neue Begriffsbenennung erfordern würde. Es ist möglich, dass nach gehöriger Einübung auch dieser Component der Empfindung zur Orientirung dienen kann. Bewegungen des Kopfes fördern die Orientirung nicht:

Eine Stunde später hat sich bei einem Versuch mit 2,5\% iger Lösung bei $37^{\circ} \mathrm{C}$. Keine specifische Empfindung gezeigt. Dagegen war eine verschwommene Geschmacksempfindung zu beobachten.

Fine halbe Stunde später führte die Wiederholung des Versuches zu unbedeutendem Schmerz, ohne dass indess Riechempfindung hätte constatirt werden können. Das Riechorgan kann den Reiz in dieser Form so zu sagen nicht verarbeiten; die Flüssigkeit ist nur bezüglich des Geschmacksorgans ein vortheilhaftes Vehikel des Reizes.

4. Mai 1902. I. Versuch mit $2,5 \%$ iger Lösung, bei $18^{\circ} \mathrm{C}$., bei vornübergebeugtem Oberkörper. Keine neue Beobachtung. Auch Kopf bewegungen ohne Resultat.

II. Versuch. 2,5\% ige Eau de Cologne-Lösung bewirkte bei $85^{\circ}$ C. zu Beginn des Eingiessens (Nasenhöhle nicht vorbereitet) eine Geruchsempfindung; im weiteren Verlaufe der Anfüllung der Nasenhöhle verschwand diese Empfindung vollkommen.

III. Versuch. 2,5\% ige Lösung bei $35^{\circ} \mathrm{C}$. in der Rückenlage; Kopf rückwärts herabhängend, ohne Vorbereitung der Nasenhöhle eingeführt. Geruchsempfindung nur während des Einströmens.

10. Mai 1902. $3 \%$ ige Eạu de Cologne-Lösung yon $34^{\circ} \mathrm{C}$., vornübergebeugter Oberkörper; unangenehmere Wirkung als bei der 2,5\% igen Lösung, aber keine specifische Empfindung. Oefters Niessen.

2 Stunden später, Versuch mit verdünnter Alkohollösung (3 Theile 90\% iger Alkohol auf 97 Theile 0,9\% ige NaCl-Lösung); die entstandene Empfindung unterscheidet sich genau von der gemischten Empfindung, welche Eau de Cologne auszulösen pflegt. Die Art der Reizung der Nervenapparate des N. trigeminus ist charakteristisch und gut unterscheidbar, z. B. ron der durch Nelkenöl bedingten Tastempfindung.

17. Mai 1902. Versuche mit 3\% iger Eau de Cologne-Lösung bei 16,5, 37 und $40^{\circ}$ C. Keine specifische Empfindung.

5. Juni 1902. Versuch mit 3\% iger Eau de Cologne-Lösung bei $21^{\circ} \mathrm{C}$. Selbst direct applicirt, ist die Flüssigkeit als bekannt yorgekommen, obwohl 
dieser neue Versuch nach längerer Pause vorgenommen worden war. Bei $40^{\circ} \mathrm{C}$. Schmerzgefühl.

6. Juni 1902. Die Nase wird mit $32^{\circ} \mathrm{C}$. warmer, $0,925 \%$ iger NaCl-Lösung vorbereitet und hierauf 2,5\% ige Eau de Cologne-Lösung eingeführt. Es entsteht zunächst ein sehr unbestimmter Reizzustand des Geruchsorganes, welcher später bei zunehmender Concentration des Riechstoffes intensiver und leichter wahrnehmbar wird; schliesslich stellt sich Schmerz ein, welcher langsam jeden anderen Reizzustand unterdrückt.

Weiterer Versuch an der rechtsseitigen Nasenhälfte mit 2,5\% iger, auf $35^{\circ} \mathrm{C}$. erwärmter Eau de Cologne-Lösung, bei vornübergebeugtem Oberkörper, nach Vorbereitung der Nasenhöhle. Bei entsprechender Concentration tritt eine combinirte Empfindung ein, bei deren Zusammensetzung auch ein specifischer Reizzustand betheiligt ist. Dieser Reizzustand hat eine vom reinen Geruchsgefühl abweichende eigenartige Färbung.

25. Juni 1902. Versuch nach H a y craft's Methode, mit 2,5\% iger Lösung, auf $30^{\circ} \mathrm{C}$. erwärmt. Auf Grund früherer Erfahrungen, wiewohl etwas unbestimmt, aher dennoch erkennbar. Nach dem Versuch war es schwer, das Kölner Wasser beim Riechen an der Luft zu erkennen.

27. Juni 1902. Die erwärmte riechende Flüssigkeit bei vornübergebeugtem Oberkörper in die Nase direct eingeführt. Die Lösung wurde von einem Assistenten in einer Pipette mir in die Hand gegeben, ohne dass ich gewusst hätte, was für ein Riechstoff es sei. Zu Beginn des Einströmens tritt eine ziemlich lebhafte gemischte Empfindung auf, welche jedoch nach Beendigung der Anfüllung der Nasenhöhle verschwindet. Es war nicht möglich, genau zu bestimmen, ob es sich um Ylang-ylang- oder Eau de Cologne-Lösung handle.

Bezüglich aller anderen geprüften Riechstoffe, sowohl an bei mir selbst, als auch an Anderen angestellten Versuchen, wurden die Aufzeichnungen im Ganzen und Grossen in ähnlicher Weise gemacht.

\section{Zusammenfassung der Versuchsergebnisse und Erwägung der Möglichkeit, in Wasser zu riechen.}

Bei Erwägung der directen Wirkung riechender Flüssigkeiten muss in Betracht gezogen werden, dass das Geruchsorgan gegen das Eindringen von Flüssigkeiten ziemlich gut geschützt ist. Wenn daher ein Reiz von diesem Aggregatzustande das Riechorgan erreichen kann, so können einerseits einzelne der Reizcomponenten sowohl die Umgebung des Riechorgans, als auch dasselbe selbst reizen, andererseits ạber können dieselben Componenten oder andere die Empfindlichkeit verändern.

Wenn auch osmotische Einflüsse und Temperaturwirkung ausgeschlossen sind, so verbleiben noch immer als Bestandtheile des 
sehr zusammengesetzten Reizes: Die mechanische Wirkung der Flüssigkeit, die specifische Reizwirkung auf das Geruchsorgan und auch auf die Nervenapparate des $N$. trigeminus, ferner die Veränderung der Empfindlichkeit in Folge der starken Durchfeuchtung und der specifischen Reizwirkung.

Wird der Versuch mit körperwarmer isotonischer $\mathrm{NaCl}$-Lösung begonnen, so verspüren wir keinen Schmerz, wenn wir die Flüssigkeit behutsam einführen. Es treten indess gewisse Paraesthesien auf, welche gewissen, aus dem Kreise der Geruchs- und Geschmacksempfindungen in ziemlich launenhafter Weise ausgewählten Qualitäten entsprechen. Aehnliche Empfindungen pflegen in manchen Fällen den Schnupfen zu begleiten. Wenn die Kochsalzlösung gewisse Gebiete, so z. B. die oberen hinteren Theile der Riechspalte oder überhaupt der Nasenhöhle erreicht, so ruft sie öfters Ekel hervor, so dass man, insbesondere wenn die Flüssigkeit in der Nasenhöhle in Bewegung kommt oder aber während des Herausfliessens derselben, leicht zusammenschauert. Die erwähnten Theile stellen jenes Gebiet vor, auf welches $Z$ w a a $\mathbf{r}$ d e maker ${ }^{1}$ ) die Zone der "Odores nauseosi“, der ekelhaften Gerüche verlegt. Die beobachtete Erscheinung kann daher auch ein Resultat der mechanischen Reizung dieses Gebietes sein. Die isotonische, an der Luft geruchlose Flüssigkeit vermag. also vielleicht schon zu Folge ihres Aggregatzustandes einen gewissen Reiz auszuüben.

Mit an der Luft geruchlosen isotonischen Lösungen gewisser mineralischer Salze können auch solche Wirkungen erzielt werden, welche als heterologe Reizungen des Riechorgans selbst betrachtet werden müssen. Aronsohn ${ }^{2}$ ) erwähnt betreffs der $4,38 \%$ igen $\mathrm{MgSO}_{4}$-Lösung und der $1,46 \%$ igen $\mathrm{NaHCO}_{3}$-Lösung als in osmotischer Beziehung dem physiologischen Kochsalzwasser äquivalenten Flüssigkeiten, dass dieselben einander gleichende Geruchsempfindungen hervorrufen; diese hat er indess nicht näher beschrieben.

Aronsohn's Beobachtung kann ich auf Grund meiner mit diesen Lösungen angestellten Untersuchungen bekräftigen. Diese Flüssigkeiten, insbesondere die $\mathrm{NaHCO}_{3}$-Lösung, erwecken eigentümliche, bald an den Geruch des verpufften Schiesspulvers, bald an den des Schwefelhydrogens erinnernde Geruchsempfindungen. An der

1) 1. c. S. 262 u. 263 .

2) 1. c. S. 334 u. 336 . 
Luft berochen, .haben diese Lösungen keinen, insbesondere keinen den eben erwähnten ähnlichen Geruch; der durch die directe Einwirkung dieser Flüssigkeiten auf die $\mathrm{R}$. olfactoria hervorgerufene Reizzustand kann daher als Parosmie angesehen werden. Schon diese Erscheinung an und für sich muss uns bei der Beurtheilung der directen Wirkung riechender Flüssigkeiten zur Vorsicht mahnen. Diese Wirkung kann in Form von Auslösung parosmatischer Empfindungen zum Ausdruck kommen, welche bei der Untersuchung der directen. Wirkung riechender Flüssigkeiten als Versuchsfehler eine Rolle spielen können.

Wenn mit irgend einer riechenden Flüssigkeit Versuche angestellt werden, so ändert sich das Resultat je nach der Kopfhaltung. und je nach dem Umstande, ob die riechende Flüssigkeit direct oder nur nach vorhergehender Vorbereitung der Nasenhöhle mit Kochsalzlösung in diese eingeführt wird.

Wird die riechende Flüssigkeit direct in die Nase gebracht, so entsteht meistens zu Beginn des Einströmens derselben eine Geruchsempfindung, welche Deutungen bezuglich der Qualität des Versuchsstoffes zulässt. Der Grund hierfür ist einfach darin zu suchen, dass Verschiebungen der Berührungsgrenzen der Flüssigkeit und der Luft, ferner zu Folge des Kampfes der Luft mit der Flüssigkeit entstehende Luftwirbel, das Geruchsorgan in gewohnter Weise reizen. Diese Geruchsempfindung stumpft sich jedoch bald $a b$ und versehwindet schliesslich beiın vollständigen Eindringen der Flüssigkeit, vorausgesetzt, dass die Haltung des Kopfes dem vollen Ueberschwemmen des Riechgebietes keine Hindernisse in den Weg setzte. Die Möglichkeit des Auftretens eines Nachgeruches ist jedoch nicht ausgeschlossen.

Aus den eben besprochenen Gründen können also zwei Umstände za Irrthümern Veranlassung geben. Vor Allem kann nämlich leicht die Meinung entstehen, dass die Riechspalte bereits vollständig überschwemmt ist, während doch in Wirklichkeit hierzu noch gar keine Möglichkeit gegeben war. In solchen Fällen wird die Geruchsempfindung in gewöhnlicher Weise durch gasartige Stoffe ausgelöst; es wäre daher ein Irrtbum, wollte man das Zustandekommen der Empfindung auf einen, durch die Flüssigkeit direct ausgelösten Reizzustand zurückführen.

Der zweite leicht irreführende Umstand ist die Möglichkeit, dass $\mathrm{zu}$ der vorausgegangenen Geruchsempfindung während der Anfüllung 
der Nasenhöhle mit Flüssigkeit sich eine Nachempfįdung zugesellt. Diese letztere kann irrthümlich für eine in Folge einer gleichzeitigen Reizung entstandene wahre Geruchsempfindung gehalten werden; sie ist jedoch vielleicht nichts Anderes, als das Nachllingen des durch die vorangegangene homologe Reizung ausgelösten Reizzustandes.

Nachdem, wie schon früher bewiesen, in der von A ronsohn angewendeten Stellung das Riechorgan nicht vollständig überschwemmt werden kann, erscheint es wahrscheinlich, dass die soeben besprochenen Erscheinungen Aronsohn zur Verwerfung des Weber'schen Satzes geführt haben ${ }^{1}$ ).

V a schide's $\cdot$ Verfahren $^{2}$ ) gibt gleichfalls keine genügende Ueberzeugung dessen, dass bei seinen Versuchen die Riechspalte vollständig überschwemmt worden war. Es scheint nämlich, dass Vaschide sich auch mit dem theilweisen Untertauchen der Riechspalte begnügt habe. So schreibt er z. B.: „Dans nos expériences, outre la détermination de la topographie des cavités nasales, nous avions une preuve que la fissure olfactive était remplie d'eau, a u moins en partie, dans le fait qu'une grande partie de liquide pénétrait dans la cavité buccale". Nach Vaschide's Verfahren kann die Riechspalte kaum mit Flüssigkeit vollständig überschwemmt werden, ausser es sollte der ganze Kopf mit abwärts sehendem Schädeldach völlig unter Wasser gebracht werden. Vaschide's Beschreibung gibt indess in dieser Hinsicht keine Aufklärung. Wird die Nase in die Flüssigkeit getaucht, so dringt die eingesogene Flüssigkeit auf dem geradesten und weitesten Wege durch die Choanen in die Mundhöhle, d. h. wenn nicht eben die Riechspalte der am tiefsten liegende Theil war, so sucht sich die Flüssigkeit ihren Weg eher durch den mittleren und unteren Nasengang, und

1) A ronsohn scheint nicht einen jeden seiner Versuche controlirt zu haben, er hat die Versuchspersonen gelegentlich auch sich selbst überlassen, wie das aus einer Bemerkung auf S. $328 \mathrm{l}$. c. geschlossen werden darf. Es ist nämlich dort von zwei Versuchspersonen die Rede, bei welchen das Eingiessen der riechenden Flüssigkeit ohne Erfolg war. A rons ohn entschuldigt sich gewissermaassen, indem er sagt: „dass auch die ... beiden ... wohl die in der Nase befindliche Flüssigkeit gerochen hätten, wenn sie die ihnen zusagende Concentration, Temperatur u. s. w. herauszufinden sich die Mühe gegeben hätten." Wenn jemand an sich selbst ohne Controle der Kopfhaltung experimentirt, kann er leicht in den Irrthum verfallen, den Kopf schon genügend gesenkt zu haben.

2) l. c. S. 166 . 
Ueber die Reizung des Riechorgans durch directe Einwirkung etc. 397

es kann eventuell in die Riechspalte, welche abseits von diesem geraden Wege fällt, Flüssigkeit gar nicht gelangen.

Wenn die Nasenhöhle mit physiologischer Kochsalzlösung vorbereitet wird, d. h. wenn wir während der Einführung der riechenden Flüssigkeit das durch Luftbewegung verursachte Auftreten der Geruchsempfindung vermieden haben, so ist die Möglichkeit des Entstehens eines Nachgeruches ausgeschlossen.

Sobald die riechende Flüssigkeit das die Nasenhöhle ausfüllende Salzwasser zu verdrängen beginnt, entsteht nach entsprechendem Vermischen der beiden Flüssigkeiten eine combinirte Empfindung, deren Qualität nicht genau bestimmt werden kann. Diese Empfindung erscheint uns zu Anfang, wenn uns noch die gehörige Uebung in der Beurtheilung mangelt, - gänzlich fremd und neu. Man hat den Eindruck, als wenn diese Empfindung das Resultat der Reizung eines neuen, uns bisher noch gar nicht bekannten Sinnesorganes wäre; wir können dieselbe gar nicht deuten und bei Beurtheilung der Qualität des Riechstoffes verwerthen.

An der Vermittlung dieser Empfindung nimmt vornehmlich die Innervation der $R$. respiratoria und der Riechspalte theil. Dementsprechend sind es vornehmlich gewisse Tastempfindungen und Reizzustände der $R$. olfactoria, welche als Componenten der erwähnten Empfindung betrachtet werden müssen. Ausserdem können wir manchmal auch eine sehr undeutlich ausgeprägte Geschmacksempfindung in der Gesammtempfindung entdecken, indess nur in Form einer schwachen Stimmung; diese ist nicht intensiver als jene paraesthetischen Reizzustände, welche manchmal auch in der Erinnerung flüchtig auftauchen können. Diese schwache, unbestimmte Geschmacksempfindung kann, wenn auch ganz allgemein in Voraus unberechenbarer Weise, manchmal auch dann auftreten, wenn bloss die Riechspalte und ein Theil der $R$. respiratoria überschwemmt sind, und die Flüssigkeit weder den Nasenrachenraum, noch den weichen Gaumen bereits erreicht hat, so dass von einer eventuellen directen Reizung der Geschmacksnervenapparate keine Rede sein kann. Diese Geschmacksempfindung pflegt übrigens unsere Aufmerksamkeit wenig in Anspruch zu nehmen, da die anderen Componenten der Gesammtempfindung mehr in den Vordergrund treten. Die Gesammtempfindung wird vornehmlich von Tastempfindungen beherrscht, welche aus dem Verzweigungsgebiete des zweiten 
Astes des N. trigeminus (N. ethmoidalis, N. nasopalatinus, N. septi narium u. s. w.) entspringen.

Diese von dem N. trigeminus vermittelten Empfindungen orientiren uns zu Anfang, wenn die Versuche uns noch neu sind, überhaupt gar nicht über die Qualität des verwendeten Reizmittels.

Die Tastempfindungen, welche von den verschiedenen riechenden Flüssigkeiten ausgelöst werden und die Schärfe von Schmerzempfindungen erreichen können, stehen einander sehr nahe, bis wir sie einigermaassen zu unterscheiden nicht gelernt haben. Die Fähigkeit, Stoffe, welche eine gemischte Geruchsempfindung auslösen, zu erkennen und zu unterscheiden, kann ziemlich leicht erreicht werden, da wir die combinirten Figenschaften solcher Stoffe von ihrem Beriechen an der Luft kennen, und wir, trotzdem auch die Flüssigkeit eine uns ungewöhnte Nebenwirkung hervorbringt, unsere Kenntnisse über den Stoff in kurzer Zeit zu verwerthen lernen. Dies habe ich unter Anderem bezüglich des Pfeffermünzöles beobachtet. Dieses Oel kann wegen seiner charakteristischen kühlenden Wirkung, welches auch noch auf $30^{\circ} \mathrm{C}$. erwärmte Lösungen besitzen, zu Folge des Reizzustandes der Nervenapparate des N. trigeminus selbst ohne längere Uebung erkannt werden.

Durch wiederholte tägliche Vornahme der Versuche erlangen wir bezüglich der Schätzung der vom $\mathrm{N}$. trigeminus vermittelten Empfindungen eine gewisse Uebung, daneben werden wir aber auch eines gewissen Reizzustandes der R. olfactoria gewahr. Die specifische Wirkung der riechenden Flüssigkeit steht jedoch nicht in geradem Verhältniss zu dem Molekülgehalt an riechendem Stoff derjenigen Flüssigkeitsmenge, welche eben die R. olfactoria bespült. Jedenfalls bringt die Flüssigkeit die Riechstoffpartikelchen in grösserer Dichte und Menge zur Riechgegend, als es die über dieselbe Flüssigkeit eingesogene Luft thun kann. Trotzdem aber kommen weder Intensität, noch Charakter des durch die Flüssigkeit hervorgerufenen Reizzustandes der Intensität und dem Charakter jener Empfindung nahe, welche beim Beriechen derselben Flüssigkeit an der Luft ausgelöst wird. Dieser durch die Flüssigkeit bedingte Reizzustand entspricht nicht dem wahren Begriffe der Geruchsempfindung; derselbe hat einen wenig ausgeprägten specifischen Charakter. Erst nach dem Abfliessen der Flüssigkeit und der sich anschliessenden reichlichen Verdunstung von den Wandungen der Nasenböhle erfahren wir durch das Einathmen der geschwängerten Luft, was aus unserer 
Empfindung fehlte. Durch dieses nachträgliche Riechen an der Luft gelangen wir aber - wie dies später noch erörtert werden soll nur dann zu einer klaren Gerucbsempfindung, wenn die Anfullung der Nase mit der Flüssigkeit nicht allzu lange gedauert hat.

Dieser von der Einwirkung der Flüssigkeit auf das Geruchsorgan herstammende Reizzustand ist zu Folge seiner Verschwommenheit und der Abschwächung seiner Specificität schwer zu localisiren. Das Umhertasten in der Localisation legt auch der Bestimmung des Charakters solcher Empfindungen Schwierigkeiten in den Weg. Dieser Reizzustand zeigt dennoch eine gewisse charakteristische Färbung, welche indess in gewissem Maasse auf Irradiation und auf gleichzeitige Reizung benachbarter Gebiete zurückzuführen ist. Das Auftreten dieser Färbung habe ich bei meinen Schlussfolgerungen, betreffend das vollständige Ueberschwemmtsein der Riechspalte, zur Hülfe gezogen.

Dass an dem Entstehen der durch die Berührung mit der Flüssigkeit ausgelösten Gesammtempfindung auch das Geruchsorgan bis zu einem gewissen Grade betheiligt ist, geht auch schon daraus hervor, dass die Empfindlichkeit des Geruchsorgans in Folge der Reizwirkung Abänderungen erfährt.

Wurde mit irgend einem Riechstoff, z. B. mit Ylang-ylang, der Versuch angestellt, so zeigte sich, wenn das Ueberschwemmen der Riechspalte gelungen und der erwähnte Reizzustand charakteristischer Färbung aufgetreten war, nach Abfliessen der Flüssigkeit aus der Nasenhöhle die Empfindlichkeit des Geruchsorganes als stark herabgesetzt. Dies habe ich sowohl an Anderen, als auch bei mir selbst beobachten können, vorausgesetzt, dass die Reizwirkung der Flüssigkeit genug lang, z. B. $30-40$ Secunden hindurch gedauert hat. Diese letztere Bedingung war zum Hervorrufen der Erscheinung unbedingt erforderlich. Die R. olfactoria ermüdet leicht unter der directen Einwirkung riechender Flüssigkeiten; eben desshalb musste ich in Fällen, wo die Versuchsperson nach dem Abfliessen der riechenden Flüssigkeit aus der Nase dem eben verwendeten Stoffe gegenüber ungetrübte Riechempfindlichkeit aufwies, mit Rücksicht auf die erwähnten Erscheinungen am vollen Gelingen des Ueberschwemmens der Riechspalte zweifeln. Diese Umstände bestärken mich auch im Anzweifeln dessen, dass in Vaschide's Versuchen das Riechorgan in der That vollständig überschwemmt worden wäre; noch mehr, da Vaschide angibt, dass bei seinen Versuchen nach 
dem Abfliessen der Flüssigkeit aus der Nasenhöhle die Riechempfindlichkeit keineswegs merkbar berührt erschien.

Es war ganz auffällig, dass nach Anwendung von Ylang-ylang z. B. das Erkennen der Riechstoffe gleicher Classe durch Beriechen an der Luft überhaupt schwer gelang, dagegen war nach Einwirkung der Ylang-ylang-Lösung die Empfindlichkeit für Campher und Pfeffermünzöl nicht verloren gegangen. Dem gegenüber konnte nach der Einwirkung von Campher- oder Pfeffermünzöllösungen und nachdem die Abstumpfung der Empfindlichkeit dieser Stoffe gegenüber beim Beriechen derselben an der Luft sich bereits eingestellt hat, der feine Geruch des Ylang-ylang leicht erkannt werden. Aehnliches beobachtete anch Aronsohn mit Bezug auf Campher und Eau de Cologne ${ }^{1}$ ).

Es scheint daher, dass verwandte Gruppen oder zu derselben Classe gehörige Riechstoffe ihre Reizwirkung nach ähnlichem Mechanismus ausüben, jund es ist kaum möglich, nach eingetretener Uebermüdung einen Unterschied zwischen diesen Mechanismen $\mathrm{zu}$ merken $\left.{ }^{2}\right)$.

Die Tastempfindungen und der Reizzustand des Geruchsorganes können nur nach entsprechender Uebung zur Erkennung der Riechstoffe benützt werden. Die.Wirkungsart irgend welcher riechenden Flüssigkeit muss so zu sagen zuerst ausgeforscht werden; nur dann wird man befähigt, bei einem weiteren Versucbe sich auf einzelne

1) I. c. $S .348$.

2) Von den Ermädungserscheinungen ausgehend, hält Aronsohn die Annahme verschiedener Geruchsnerven für berechtigt. Meiner Ansicht nach ist diese Folgerung noch weiter auszudehnen, indem wir annehmen müssen, dass diesen verschiedenen Nerven auch verschiedene Riechzellen entsprechen. Diese Annahme ist auch schon darum am Platze, weil, wie bekannt, der Nerv beim Mangeln eines Transformators unter den, verschiedenen Empfindungsqualitäten entsprechenden Reizwirkungen keine Auswahl treffen kann; zu einer solchen Thätigkeit ist unbedingt ein mehr ausgesprochener Zellcharakter erforderlich. Die Auswahl des zu verarbeitenden Reizes wird von den Riechzellen besorgt; diese Transformatoren, als Organe mit beibehaltenem Zellcharakter, sind der Frmüdung indess in erster Reihe ausgesetzt. Die Nerven selbst ermüden ja überhaupt nicht so leicht. Vor der Hand haben wir keinen Grund, von Unterschieden in der Structur der einzelnen Riechnervenbahnen zu reden, während bei den Riechzellen, bezüglich der mikrochemischen Reactionen und der Structur, gewisse Unterschiede glaubhaft erscheinen. Indess ohne hierauf Rücksicht zu nehmen, erscheint es aus den angeführten Gründen für richtiger, die Ermüdungserscheinungen in erster Reihe auf Veränderungen der Riechzellen zurückzuführen. 
Componenten der Gesammtempfindung zurückerinnern zu können. Ohne einer solchen Erinnerung wäre aber unsere Orientirung bei einem späteren, etwa nach einigen Tagen vorgenommenen Versuche keineswegs verlässlich.

Welche Wichtigkeit der Uebung zugemessen werden darf, das habe ich sowohl an Anderen, als auch bei mir selbst erfahren. Anfangs, in den ersten Tagen, ja in den ersten Wochen, können die Versuchspersonen, wenn die Anfüllung der Nase gut gelungen ist, überhaupt keine Rechenschaft von : den durch die Flüssigkeit ausgelösten Empfindungen geben. Eine meiner Versuchspersonen war nach einigen Wochen so weit zu bringen, dass sie nach der Anstellung von 8-10 Versuchen mit einem und demselben Stoffe angeben konnte, welcher Classe oder welcher Unterabtheilung der in der angewendeten Flüssigkeit gelöste Riechistoff hingehört, ob es ein allgemein gebräuchliches Parfum oder ätherisehes Oel u.s. w. sei. Nähere Unterscheidung indess, z. B. Festsetzung dessen, ob der verwendete Stoff Ylang-ylang, Eau de Cologne oder Essbouquet gewesen sei, war noch nicht möglich geworden; diesbezüglich konnte der Betreffende nur Muthmaassungen aufstellen.

Eine andere Versuchsperson wieder konnte selbst nach öfter wiederholten Versuchen nicht weiter als zum Wahrnehmen von Tastempfindungen gebracht werden.

An mir selbst habe ich es mit Uebung so weit gebracht, dass ich gewisse Stoffe erkannte, bevor dieselben noch in irgend einer Richtung auch nur die geringste specifische Reizwirkung ausgeübt hätten können. Selbstverständlich wurde bei derartigen Versuchen die Wahl und das Erwärmen der riechenden Flüssigkeit, deren Einfüllung in die Burette u. s. w. von Anderen besorgt, ich hielt mich während dessen in einem anderen Zimmer auf, so dass ich von der Natur des zu verwendenden Stoffes in vornherein um so weniger orientirt sein konnte, als ich bis zur Einführung der Flüssigkeit und selbstredend auch noch später durch den Mund athmete. Bei solchen Versuchen füllte ich die Nase stets vorerst mit Salzwasser an und liess die mir unbekannte riechende. Flüssigkeit erst dann vorsichtig in die Nase fliessen. Unter solchen Umständen konnte der Riechstoff zuerst nur mit der $R$. respiratoria in Berührung kommen. In ganz auffälliger Weise zeigte es sich hierbei, dass im Besitze eines gewissen Eingeübtseins schon auf Grund der Wirkung im Vestibulum einigermaassen Orientirung darüber zu gewinnen war, 
zu welcher Gruppe der Riechstoffe manche der verwendeten Riechmittel hingehörten; doch war diese Orientirung keineswegs vollständig sicher. Die bei meinen Versuchen verwendeten Riechstoffe haben sich in dieser Hinsicht in ganz charakteristische Gruppen scheiden lassen. So war es z. B. schwer, Eal de Cologne von Ylang-ylang, Campher von Pfeffermünzöl, Nelkenöl von Origanumöl zu unterscheiden. Dagegen war es sehr leicht zu bestimmen, ob es sich um Nelkenöl oder Ylang-ylang, ebenso ob es sich um Campher oder Origanumöl, um Pfeffermünzöl oder Capronsäure-Lösung gehandelt hat.

Die Einübung im Erkennen der Riechstoffe verhält sich analog der Fähigkeit der Blinden, die, wie man es gewöhnlich zu sagen pflegt, mit Hülfe ihrer häufig wiederholten Tastversuche auch auf solche Eigenschaften mancher Gegenstände kommen, welche von einem Sehenden allein durch das Betasten nicht constatirt werden könnten. Der Blindgeborene kann sich jedoch den Begriff von Farbe nicht verschaffen. Analog hierzu lassen auch die Reizzustände, welche durch in Lösungen befindliche Riechstoffe erweckt werden, die ausgebreitete Färbungscala vermissen, welche wir beim Beriechen an der Luft kennen. Sind die charakteristischen Tastempfindungen nicht so intensiv, dass sie Schmerz bedingen, so können sie, mit Hülfe ihrer Irradiation, den Reizzustand der R. olfactoria verstärken. Dem letzteren gehen jedoch gewiss viele Qualitäten der reinen Geruchsempfindung und vornehmlich deren entsprechende Schärfe ab. Wenn aber aus einer zusammengesetzten Empfindung der eine oder der andere ihrer bekannten Componenten zur Geltung kommen kann, so vermag die Thätigkeit des Gehirns aus diesem Theil der Empfindung die Gesammtempfindung selbst aufzubauen. Dies kann vornehmlich im Functionskreise des Geruchssinnes geschehen, bei welchem Associationen mit besonderer Vorliebe und Leichtigkeit aufzutauchen pflegen. Bei der Auslösung solcher Associationsprocesse können vielleicht auch die Tastgefühle betheiligt sein.

Der specifische Component der Gesammtempfindung pflegt keine dem wahren Begriffe der Geruchsempinindung entsprechende Reinheit aufzuweisen. Eine derartige Empfindung dürfte dem Beute oder Nahrung u. s. w. suchenden oder dem fliehenden Thiere nicht von grosser Hülfe sein. Die Gesammtempfindung darf mit Rücksicht auf die Schärfe des specifischen Componenten einer solchen Geschmacksempfindung verglichen werden, zu welcher sich keine Geruchs- 
empfindung gesellen kann. Wenn man beim Verkosten irgend einer Speise die Nase zuhält, so dass aus dem Munde und dem Rachen die mit Riechstoffen beladene Luft nicht von rückwärts her durch die Nase dringen könne, so verliert die bis dahin noch sehr gemischte Empfindung sofort vieles an Qualität und Färbung und wird sehr einförmig.

Zur Unterstützung des mit Bezug auf die Rolle des N. trigeminus bisher Angeführten soll noch bemerkt werden, dass die vermittelnde Thätigkeit dieses Nerven, wie bekannt, auch beim Beriechen mancher Stoffe an der Luft von Bedeutung ist. Chlor, Brom und andere Gase, sowie auch im Allgemeinen Stoffe von widerlichem Geruch, nehmen bei der Entfaltung ihrer Wirkung, zu Folge deren dieselben als eine besondere Gruppe von Riechstoffen betrachtet zu werden pflegen, - auch die Vermittlung des N. trigeminus in Anspruch. Die Orientirungsfähigkeit für diese Gerüche bleibt auch nach Lähmung des N. olfactorius bestehen, ja diese Fähigkeit kann selbst nach Lähmung des Geruchsnerven noch erlangt werden. Daher stammt wohl die irrige Meinung, dass die Zerstörung des N. olfactorius die Möglichkeit des Riechens noch nicht ausschliesst. Die Orientirung für einzelne Gerüche mag in solchen Fällen auf Einübung von solchen Empfindungen zurückzuführen sein, welche vom $\mathrm{N}$. trigeminus vermittelt wurden; eine wirklich reine Geruchsempfindung kann indess natürlich nicht mehr zu Stande konımen.

Gewisse Riechstoffe, welche durch Vermittlung des N. trigeminus gemischte Empfindungen auslösen, haben auch auf den Organismus eine tiefdringende Wirkung. Eine solche ist z. B. die Wirkung auf das Athmen, wie das aus Kratsehmer's') und H. Be yer's ${ }^{2}$ ) Versuchen hervorgeht. Nach Beyer wirkte Moschustinctur auf die Athmungsbewegung von Kaninchen viel schwächer, wenn der N. trigeminus durchgeschnitten wurde; Essigsäure entfaltet eine besonders starke Wirkung nach Durchschneidung des N. olfactoriusu.s. w. Diese Erscheinungen beweisen, dass der N. trigeminus an dem Verarbeiten der Riechreize, je nach der Art des Reizes, mehr - weniger Theil nimmt. Diese Betheiligung wird indessen nur durch Reizung

1) Ueber Reflexe der Nasenschleimhant auf Athmung und Kreislauf. Sitzungsberichte d. kais. Akad. d. Wissensch. zu Wien Bd. 62 Th. 2. 1870.

2) Athemreflexe auf Olfactoriusreiz. Arch. f. (Anat. u.) Physiol. 1901 H. 3-4. S. 261. 
grosser empfindlicher Flächen möglich. Selbst wenn man im Verlaufe einer längeren Reihe von Versuchen mit riechenden Flüssigkeiten schon ziemliche Uebung erlangt hat, bleibt die durch den N. trigeminus vermittelte Empfindung dennoch stets unbestimmt, und gibt bezüglich des Erkennens des Riechstoffes keine gehörige Orientirung, wenn z. B. nur das Vestibulum der Nase dem Reize ausgesetzt wird.

An der Hand eines gewissen, bereits erworbenen Grades von Eingeübtsein ist es möglich, einige grössere Gruppen der Riechstoffe von einander zu unterscheiden. Unsere Orientirung ist jedoch nur zum geringen Theile der Function des Geruchsorganes zuzuschrëiben. Die Färbung des specifischen Reizzustandes erleidet eine Veränderung. Der in irgend einer Flüssigkeit aufgelöste riechende Stoff büsst von seinen charateristischen Eigenschaften ein, und es geht ihm die Hauptbedingung seiner Wirkung, nämlich die Energie der Verdunstung und der Diffusion ab.

Bei den meisten meiner Versuche war ich bemüht, die Wirkungsart riechender Luft wenigstens theilweise nachzuahmen, indem ich die Flüssigkeit, wie es im III. Capitel beschrieben wurde, in Bewegung brachte. Das Resultat war, dass die Orientirung nư in spärlichen Fällen eine bestimmtere Richtung erhielt. Da die Reizwirkung der sich bewegenden Flüssigkeit theilweise den bei homologer Reizung des Geruchsorganes stattfindenden Mechanismus nachahmt, so könnte man erwarten, dass der Reizzustand in Folge der Bewegung der Flüssigkeit selbst dann intensiver werden sollte, weńn das Mitreissen von Luftbläschen ausgeschlossen werden kann. Diese Verstärkung: des Reizzustandes tritt jedoch überhaupt nicht regelmässig auf. Die Bewegung solcher riechender Flüssigkeiten, bei welchen die Auflösung des Riechstoffes eine unvollkommene war, entfaltet noch am ehesten eine Wirkung. Pfeffermünz-, Nelken- und Origanumöl geben mit Salzwasser theilweise Emulsion; während des Strömens dieser Flüssigkeit können sich bewegende Oelkügelchen mit der Oberfläche der Riechspalte in Berührung kommen, so dass vielleicht auch der Unterschied in der Oberflächenspannung zur Geltung kommen mag.

Eine weitere Frage ist nun, ob die Geruchsempfindung eine Veränderung erfährt, wenn die Riechstoffpartikelchen in Dampf oder Dunst gehüllt zu dem Geruchsorgan gelangen. Zur Klärung dieser Frage habe ich mit Nelkenöl-, Campher- und Ylang-ylang-Lösungen im 
Dampfbad von $+45^{\circ} \mathrm{G}$. Versuche vorgenommen. Die Lösungen habe ich aus Fläschehen von $50 \mathrm{ccm}$ Inhalt gerochen, welche eine Oeffnung von $12-13 \mathrm{~mm}$ Weite hatten. Es zeigte sich, dass der specifische Charakter anfangs keine besondere Veränderung erlitt, und sich nur nach einiger Zeit abstumpfte, dann nämlich, wenn schon auf allen Theilen der Nasenhöhle Niederschläge des Wasserdampfes sassen, gewiss also auch die Cilien der Riechzellen einen solchen Belag. aufwiesen. Betreffs der in Frage kommenden Riechstoffe war nur die Veränderung zu merken, dass die Fähigkeit, sich auf grössere Entfernung fühlbar zu machen, d. h. das Diffusionsvermögen geschwächt war, obwohl die erhöhte Temperatur $\left(45^{\circ} \mathrm{C}\right.$.) für das Penetrationsvermögen eigentlich eine günstige Bedingung abgeben sollte. In einer mit sichtbarem Dunst erfültten Atmosphäre wird der Geruch einer riechenden Flüssigkeit, welche wir an unseren Handflächen zerreiben, aus einer Entfernung von nur $20-30 \mathrm{~cm}$, nur sehr spät oder gar nicht fühlbar, während doch in einer Atmosphäre mit normaler Feuchtigkeit die Geruchsempfindung bei solcher Distanz sehr bald einzutreten pflegt. Dieser Versuch muss daher den Gedanken erwecken, dass in einer mit Wasserdunst oder -Dampf gesättigten Atmosphäre die Riechpartikelchen bei ihren Bewegungen stets auf Hindernisse stossen; und dass diese Hindernisse eventuell genügen, um in einiger Entfernung von der Verdunstungsfläche die Bewegung der riechenden Partikelchen zu unterdrücken ${ }^{1}$ ).

In anderen Fällen habe ich in Zimmerluft von normaler Feuchtigkeit die Wirkung der aus den riechenden Lösungen entsteigenden sichtbaren Dünste und Dämpfe in Beobachtung gezogen. Diesbezüglich war im zweiten Abschnitt des III. Capitels schon Einiges erwähnt. Lässt man die Flüssigkeit in einer Eprouvette aufkochen und beriecht dann die während des Kochens aufsteigenden Dämpfe, so schwächt sich die reine Geruchsempfindung ab, und die Gesammtempfindung wird in ganz auffallender Weise jener Empfindung ähn-

1) Die von Aronsohn bezüglich der Rolle des Wassers beim Riecheñ erwähnte Erscheinung, dass nämlich die Blätter gewisser Kräuter, sowie auch bituminöse Mineralien nur nach Benetzung einen Geruch verbreiten, hat keinen Bezug auf das eben Gesagte und kann nicht zur Anfechtung des W eber'schen Satzes dienen. In Folge der Wirkung des. Wassers kann in den Kräuterblättern Hydrolyse oder Oxydation, bei den Mireralien vornehmlich Oxydation u. s. w. erfolgen: diese chemischen Processe können dann zur Entstehung des Riechstoffes führen. ( $\mathrm{Z}$ w a ardemaker.) 
lich, welche die Flüssigkeit selbst; bei Einführung in die Nasenhöhle, auszulösen pflegt. Wenn es sich um Stoffe handelt, welche schon unter normalen Verhältnissen die Nervenapparate des N. trigeminus zu reizen im Stande sind, wie z. B. das Pfeffermünzöl, so wird die Wirkung der heissen Dämpfe ungemein stark; es können auch sehr breit ausstrahlende, weitgehende Reflexe auftreten. Unter solchen Umständen nimmt das Pfeffermünzöl, welches von $\mathrm{Z}$ w a a r d e $\mathrm{m}$ a $\mathrm{k}$ e r zu der Unterabtheilung $\mathrm{C}$ der Classe II. zugezählt wird, solche Eigenschaften an, welche diesen Stoff eher den sog. widerlichen Riechstoffen angliedern.

Mit der Abkühlung kehren die ursprünglichen Eigenschaften der Lösung wieder zurück; die Intensität des von ihr verbreiteten Geruches ist kaum, ihr specifischer Charakter gar nicht geschwächt.

Die Beweglichkeit der riechenden Partikelchen wird in sichtbaren Wasserdämpfen schwächer; im Wasser kommt sie gar nicht mehr zur Geltung. Die Bewegung kann nur von den Berührungsflächen der Flüssigkeit und der Luft ausgehen, wie dies die bekannten eigenthümlichen Bewegungen von Camphertheilchen auf der Wasseroberfläche zeigen. Der Campher muss indess an die Oberfläche des Wassers kommen, um sich bewegen zu können. Fin in die Flüssigkeit versunkenes Riechpartikelchen hat daher mit Bezug auf das Geruchsorgan nur eine potentielle Energie.

Der Riechstoff kann also, wie dies aus dem Charakter der ausgelösten Gesammtempfindung, sowie aus den Verhältnissen des Aggregatzustandes übereinstimmend folgt, in Form von Flüssigkeit in die Riechspalte gebracht, nur als heterologer Reiz des Geruchsorgans wirken.

Von heterologen Reizen ausgelöste Empfindungen können zur Erklärung der Thätigkeit irgend eines Sinnesorgans nicht benützt werden. Es wäre daher nicht zweckmässig, den E. H. W eb er'schen Satz, welcher zur Erklärung des reinen Begriffes der Geruchsempfindung dient, zu verwerfen, wenn auch Weber zu der Aufstellung desselben an der Hand von unter keineswegs günstigen Bedingungen angelegten Versuchen gelangt war.

Der das Geruchsorgan dick überziehende Schleim legt den Riechpartikelchen Hindernisse in den Weg. Es bedarf besonderer Einrichtungen, damit der Reiz durch diesen dicken Schleim bis zum 
Ueber die Reizung des Riechorgans durch directe Einwirkung etc. 407

Geruchsorgan vordringen könne. Darauf weisen wenigstens die von $\mathrm{J}$ ag $0 \mathrm{dowski}^{1}$ ) an Hechten vorgenommenen histologisehen. Untersuchungen. Nach Jagodowski sind die in den Falten der Riechschleimhaut tiefliegenden Riechzellen des Hechtes von einer derart dicken Schleimschicht bedeckt, dass der Reiz kaum zu den Riechzellen gelangen könnte, wenn die "Riechgeisseln", welche er als Nervenfäden betrachtet, nicht die Uebertragung des Reizes möglich machen würden.

Bei den Wasserthieren kann von einer Geruchsempfindung in dem Sinne, wie bei den makrosmatischen Thieren, keine Rede sein. Bezüglich der Beurtheilung der "Geruchsempfindung" der Ersteren mag es genügen, auf die Untersuchungen W. $\mathrm{Nagel}^{\prime} \mathrm{s}^{2}{ }^{3}$ ) hinzuweisen, der seine Auffassung der Frage durch Aufstellung des Begriffes des "chemischen Sinnesorganes" ausdrückt.

Es soll hier bemerkt werden, dass die von Aronsohn bezüglich der "Geruchsempfindung" der Fische angestellten Versuche von einer derartigen Fähigkeit dieser Thiere nicht überzeugen können. Um das Riechvermögen der Fische zu prüfen ${ }^{4}$ ), hat er Goldfischen Ameiseneier gereicht, welche mit Nelkenöl oder Tinctura asae foetidae stark durchgetränkt waren. Nach Berühren dieser sonst gerne genommenen Beute entfernten sich die Fische rasch von derselben. A rousohn folgerte aus dieser Erscheinung, dass das Geruchsorgan der Thiere eine Reizung erfahren habe, was er übrigens auch dadurch bewiesen meinte, dass die Fische nicht nur nach der Berührung der Lockspeise, sondern öfters schon in einer Distanz von einigen Millimetern zu dieser Kehrt machten.

Aus diesen Erscheinungen könnten wir indess mit demselben Rechte auf Tastempfindlichkeit schliessen, wie dies Aronsohn mit Bezug auf die Geruchsempfindlichkeit gethan. Das aus den Ameiseneiern herausdiffundirende, die Lockspeise einige Millimeter breit umgebende Nelkenöl (oder der Alkohol) kann auch durch Vermittlung der Tastorgane Vertheidigungsreflexe erwecken. Wenn z. B. auf die Haut eines Frosches in der Umgebung des Afters $0,1 \%$ ige Nelkenöl-

1) Zur Frage nach der Endigung des Geruchsnerven bei den Knochenfischen. Anat. Anz. Bd. 19 (11) S. 257. 1901.

2) l. c.

3) Versuche zur Sinnesphysiologie von Beroë ovata und Carmarina hastata. Pflüger's Arch. f. d. ges. Physiol. Bd. 54 S. 165. 1893.

4) $1 . \mathrm{c}$. 
408 Flemér Veress: Ueber die. Reizung des Riechorgans etc.

lösung oder nur schwachriechende Vanillinlösung aufgetropft wird, reibt er mit seinen Füssen die Flüssigkeit erregt von sich ab, gerade so, als ob wir eine Säure auf seine Haut gebracht hätten.

$\mathrm{Nagel}$ erwähnt vom. Dytiscus marginalis, dass dieser. Käfer Fleisch auf eine grössere Entfernung als $1 \mathrm{~cm}$ nicht mehr wahrninimt. Die von Aronsohn beobachteten Fische bemerkten die widerlichen Eigenschaften ihrer Speise nur in einer Entfernung von einigen Millimetern. Einem Sinnesorgan, welches ein Reizmittel nur aus so geringer Entfernung wahrnimmt, fehlen die charakteristischen Eigenschaften eines selbstständigen Geruchsorgans, d. h. die Fähigkeit, gewisse Stoffe aus grosser Entfernung zu erkennen. Diese Fähigkeit kann nur beim Riechen an der Luft zur Geltung kommen. 